\title{
QCD calculations of radiative heavy meson decays with subleading power corrections
}

\author{
Hua-Dong Li, ${ }^{a}$ Cai-Dian Lü, ${ }^{a, b}$ Chao Wang, ${ }^{c}$ Yu-Ming Wang ${ }^{c}$ and Yan-Bing Wei ${ }^{c}$ \\ ${ }^{a}$ Institute of High Energy Physics, \\ CAS, P.O. Box 918(4) Beijing 100049, China \\ ${ }^{b}$ School of Physics, University of Chinese Academy of Sciences, \\ Beijing 100049, China \\ ${ }^{c}$ School of Physics, Nankai University, \\ Weijin Road 94, 300071 Tianjin, China \\ E-mail: lihd@ihep.ac.cn, lucd@ihep.ac.cn, chaowang@nankai.edu.cn, \\ wangyuming@nankai.edu.cn, weiyb@nankai.edu.cn
}

ABSTRACT: We revisit QCD calculations of radiative heavy meson decay form factors by including the subleading power corrections from the twist-two photon distribution amplitude at next-to-leading-order in $\alpha_{s}$ with the method of the light-cone sum rules (LCSR). The desired hard-collinear factorization formula for the vacuum-to-photon correlation function with the interpolating currents for two heavy mesons is constructed with the operatorproduct-expansion technique in the presence of evanescent operators. Applying the background field approach, the higher twist corrections from both the two-particle and threeparticle photon distribution amplitudes are further computed in the LCSR framework at leading-order in $\mathrm{QCD}$, up to the twist-four accuracy. Combining the leading power "pointlike" photon contribution at tree level and the subleading power resolved photon corrections from the newly derived LCSR, we update theory predictions for the nonperturbative couplings describing the electromagnetic decay processes of the heavy mesons $H^{* \pm} \rightarrow H^{ \pm} \gamma$, $H^{* 0} \rightarrow H^{0} \gamma, H_{s}^{* \pm} \rightarrow H_{s}^{ \pm} \gamma$ (with $H=D, B$ ). Furthermore, we perform an exploratory comparisons of our sum rule computations of the heavy-meson magnetic couplings with the previous determinations based upon different QCD approaches and phenomenological models.

KEYWORDS: NLO Computations, QCD Phenomenology

ARXiv EPrint: 2002.03825 


\section{Contents}

1 Introduction 1

2 Theory summary for radiative heavy meson decays 5

3 The twist-two LCSR for the resolved photon effect 10

$\begin{array}{ll}3.1 \text { Hard-collinear factorization at LO in QCD } & 10\end{array}$

3.2 Hard-collinear factorization at NLO in QCD 12

4 The higher twist LCSR for the resolved photon effects 18

$\begin{array}{lll}4.1 & \text { Two-particle higher twist corrections } & 19\end{array}$

4.2 Three-particle higher twist corrections 20

5 Numerical analysis $\quad 23$

5.1 Theory inputs 23

5.2 Theory predictions for radiative heavy meson form factors 25

6 Conclusion 28

$\begin{array}{ll}\text { A Useful one-loop integrals } & 30\end{array}$

B Master formulae for the spectral representations 31

$\begin{array}{ll}\text { C Photon distribution amplitudes } & 32\end{array}$

\section{Introduction}

Advancing our understanding of radiative heavy meson decays is, on the one hand, of importance for exploring the emerged symmetries of the QCD Lagrangian in the limit of massless light quarks and of infinitely heavy quarks, on the other hand, crucial to develop a systematic formalism for computing the electromagnetic corrections to flavour-changing weak decays of heavy hadrons, which are indispensable for a detailed anatomy of the quark flavour dynamics of the Standard Model (SM). On the phenomenological aspects, precision calculations of radiative heavy meson decays are also in high demand for the sake of determining the magnetic susceptibility of the quark condensate $\chi(\mu)$ [1], which parameterizes the response of the QCD vacuum with respect to an external electromagnetic field and serves as an essential nonperturbative input for the theory description of exclusive hadronic reactions involving on-shell photons [2-12] and for the improved calculations of the muon anomalous magnetic moment [13]. Consequently, distinct QCD techniques have been developed to allow for the systematical computations of radiative heavy meson decay amplitudes based upon the heavy quark expansion technique and perturbative factorization theorems. 
Employing the method of the two-point QCD sum rules (QCDSR), the electromagnetic $D^{*}(p+q) \rightarrow D(q) \gamma(p)$ decay form factors were estimated at leading-order (LO) in $\alpha_{s}$ [14] by taking into account the subleading power corrections at the dimension-5 quark-gluon condensate accuracy. However, implementing an approximation to avoid the double Borel transformation with respect to the variables $p^{2}$ and $(p+q)^{2}$ [14] yields contamination of the obtained sum rules due to the "non-diagonal" transitions of the ground state to excited states. The resulting prediction for the branching fraction of $D^{* 0} \rightarrow D^{0} \gamma$ turned out to be larger than that for the strong decay process $D^{* 0} \rightarrow D^{0} \pi^{0}$ [14], in contradiction with the experimental measurements from the CLEO [15, 16], BaBar [17], and BES III [18] Collaborations. Subsequently, the radiative charm-meson decay form factors were computed from the three-point QCDSR approach, ${ }^{1}$ including the power suppressed corrections from the higher-dimension operators up to the four-quark condensate [20], yielding the theoretical predictions in reasonable agreement with the experimental results [15-18]. As demonstrated in [20], the numerically dominant contribution to the tree-level sum rules for the radiative $D_{(s)}^{*+} \rightarrow D_{(s)}^{+} \gamma$ form factors arises from the dimension-3 quark condensate correction instead of the leading power perturbative effect, due to the strong cancellation for the photon radiation off the charm and the anti-down (anti-strange) quarks, which also justifies the high suppression of the $D_{(s)}^{*+} D_{(s)}^{+} \gamma$ coupling compared with the magnetic moment for the counterpart neutral $D^{* 0}$-meson. In an attempt to eliminate the substantial contamination from the non-diagonal transitions of constructing the traditional QCDSR for hadronic matrix elements at small momentum transfer, the technically improved sum rules based upon the light-cone operator-product-expansion (OPE) for the corresponding QCD correlation functions have been constructed [21] for computing the radiative heavy meson decay form factors with the subleading power corrections from the photon light-cone distribution amplitudes (LCDA) at the (partial)-twist-four accuracy. Motivated by the systematic classification of the two-particle and three-particle photon distribution amplitudes with the background field formalism [22], the updated light-cone sum rules (LCSR) for the electromagnetic heavy-meson decay form factors were further derived in [23] including the two-particle and three-particle "hadronic" photon corrections at twist-four completely.

Taking advantage of the spontaneously broken chiral symmetry for the light quarks and the emerged spin-flavour symmetry for the heavy quarks, systematic computations of the radiative heavy-meson decay form factors have been also carried out in the framework of heavy hadron chiral perturbation theory $(\mathrm{HH} \chi \mathrm{PT})[24-26]$, including the one-loop corrections to symmetry breaking effects at $\mathcal{O}\left(m_{q}\right)$ and $\mathcal{O}\left(1 / m_{Q}\right)[27]($ see $[28,29]$ for further discussions). The striking large SU(3)-flavour symmetry breaking effects for the $D_{q}^{*} \rightarrow D_{q} \gamma$ (with $q=u, d, s$ ) decay rates can be apparently traced back to the numerical cancellation between the leading power and the subleading power contributions in the heavy quark expansion, by accident, for the charged charm-meson form factors with physical values of the charm-quark mass. However, the two effective parameters $g_{\pi}$ and $\beta$ characterizing the $M^{*} M \pi$ and $M^{*} M \gamma$ couplings (with $\left.M=D_{(s)}, B_{(s)}\right)$ can only be extracted from the avail-

\footnotetext{
${ }^{1}$ The double dispersion sum rules for computing radiative transition form factors in QCD were originally suggested in [19].
} 
able experimental measurements of the corresponding vector charm-meson decays [27] or determined by the non-perturbative QCD techniques (see [30-34] and references therein), generating a significant limitation of the predictive power for the $\mathrm{HH} \chi \mathrm{PT}$ formalism. We further mention in passing that the electromagnetic transition form factors of the low-lying heavy mesons have been evaluated phenomenologically [35] by incorporating the $\mathrm{HH} \chi \mathrm{PT}$ framework and the vector meson dominance (VMD) hypothesis, which gives rise to the potentially sizeable systematic uncertainty of the resulting predictions and should be improved upon by employing the dispersion approach as developed in [36] (see [9, 37, 38] for more applications).

On account of the substantial power suppressed corrections to the radiative $D_{(s)}^{*+} \rightarrow$ $D_{(s)}^{+} \gamma$ decay form factors, it is apparently of importance to compute perturbative QCD corrections to the resolved photon contributions to all the magnetic couplings responsible for $H^{* \pm} \rightarrow H^{ \pm} \gamma, H^{* 0} \rightarrow H^{0} \gamma, H_{s}^{* \pm} \rightarrow H_{s}^{ \pm} \gamma$ (with $H=D, B$ ) at the twist-two accuracy and to refine the aforementioned higher twist hadronic photon corrections at tree level presented in $[21,23]$ by employing the LCSR method, in order to achieve a better understanding of the heavy quark expansion for the charm and bottom hadron decays and to provide the state-of-art theory predictions for the heavy-meson electromagnetic transition form factors in QCD. We summarize the essential new ingredients of the present paper as follows.

- We establish the hard-collinear factorization formula for the twist-two contribution to the vacuum-to-photon correlation function defined by the vector and pseudoscalar interpolating currents for two heavy mesons at next-to-leading-order (NLO) in QCD, with the aid of the evanescent operator approach $[39,40]$ and the strategy of regions [41, 42]. The double spectral density appearing in the dispersion representation of the obtained factorization formula will be derived analytically for the asymptotic photon distribution amplitude $\phi_{\gamma}^{\text {asy }}(u, \mu)=6 u(1-u)$, following the prescriptions introduced in [43]. In particular, we extract the general expression for the QCD spectral function of the NLO correction to the twist-two hadronic photon contribution of the vacuum-to-photon correlation function, without implementing the further reduction dependent on the specific shape of the duality region for the resulting dispersion integral [44]. QCD resummation for the enhanced logarithms of $m_{Q} / \Lambda_{\mathrm{QCD}}$ entering the hard-collinear factorization formula will be accomplished at next-to-leadinglogarithmic (NLL) accuracy by solving the renormalization-group (RG) equation of $\phi_{\gamma}(u, \mu)$ at two loops.

- We perform the continuum subtraction for constructing the sum rules of the higher twist corrections to the $M^{*} M \gamma$ couplings by computing the corresponding double spectral densities at LO in $\alpha_{s}$ analytically, instead of invoking the simple replacement rule valid for the leading twist contribution as employed in [21, 23]. Our method can be also generalized to the LCSR calculation of the $M^{*} M \pi$ couplings straightforwardly. 
- We compute the light-quark mass effect for the "point-like" photon contribution to the radiative heavy meson decay form factors at tree level and demonstrate explicitly that such $\mathrm{SU}(3)$-flavour symmetry breaking effect is power suppressed in the heavy quark expansion, in contrast to the observed pattern for heavy-to-light $B$-meson decay form factors at large hadronic recoil $[45,46]$. Furthermore, we identify the precise correspondence for all separate terms entering the "point-like" photon contributions to the $M^{*} M \gamma$ couplings between the LCSR calculation and the $\mathrm{HH} \chi \mathrm{PT}$ analysis.

This paper is structured as follows. In section 2 we will briefly review the two equivalent definitions of the heavy meson magnetic couplings responsible for the radiative $M^{*} \rightarrow M \gamma$ transitions, and then summarize the "point-like" photon contributions to the tree-level sum rules of the $M^{*} M \gamma$ couplings by exploring the factorization properties of the vacuumto-photon correlation functions under discussion in detail and computing the subleading power perturbative correction due to the non-vanishing light quark mass at LO in the strong coupling constant $\alpha_{s}$. We will proceed to establish the hard-collinear factorization formula for the twist-two resolved photon contribution to the vacuum-to-photon correlation function at one loop with the OPE technique in section 3, where the general expression of the double spectral density for the obtained QCD representation of the above-mentioned correlation function at NLO will be derived analytically and the resummation improved LCSR for the twist-two hadronic photon correction will be achieved with the standard RG resummation technique in momentum space. Employing the background field approach we will turn to compute the subleading power contributions to the radiative heavy meson decay form factors from the two-particle and three-particle higher twist photon distribution amplitudes at the twist-four accuracy within the same LCSR framework in section 4, with an emphasis on the technical derivation of the perturbative double spectral functions in the corresponding dispersion integrals. Phenomenological implications of the refined LCSR for the $M^{*} M \gamma$ couplings will be subsequently explored in section 5, by investigating the numerical impacts of the distinct subleading power contributions with seven non-perturbative parameters appearing in the conformal expansion of the collinear photon LCDA determined from the QCDSR method $[2,22]$ and by performing an exploratory comparison of our LCSR calculations with the previous determinations from the $\mathrm{HH} \chi \mathrm{PT}$ method and from the lattice QCD simulation. Section 6 will be reserved for a summary of our main observations on the resolved photon corrections and for theory perspectives on the future improvements of QCD calculations of the electromagnetic heavy meson transition form factors. We further collect the useful results of various one-loop Feynman integrals and the master formulae to derive the spectral representation of the twist-two factorization formulae for the vacuum-to-photon correlation function at NLO in appendices A and B, respectively. For completeness, we also summarize the definitions of the two-particle and three-particle collinear photon LCDA at twist-four in appendix C. 


\section{Theory summary for radiative heavy meson decays}

The purpose of this section is to present the tree-level LCSR for the "point-like" photon contribution to the $M^{*} M \gamma$ coupling including the SU(3)-flavour symmetry breaking effect due to the light-quark mass correction. The vector-to-pseudoscalar heavy meson electromagnetic transition matrix element will be parameterized in the standard way

$$
\left\langle M(q)\left|j_{\mu}^{\mathrm{em}}\right| M^{*}(p+q, \varepsilon)\right\rangle=g_{\mathrm{em}} \frac{2 i \mathcal{V}\left(p^{2}\right)}{m_{V}+m_{P}} \epsilon_{\mu \nu \rho \sigma} \varepsilon^{\nu} p^{\rho} q^{\sigma},
$$

where we have introduced the following conventions

$$
j_{\mu}^{\mathrm{em}}=g_{\mathrm{em}} \sum_{q} e_{q} \bar{q} \gamma_{\mu} q, \quad \epsilon_{0123}=-1,
$$

and $m_{V(P)}$ is the heavy vector (pseudoscalar) meson mass. The on-shell photon coupling with the vector and pseudoscalar heavy mesons defined as

$$
\left\langle\gamma\left(p, \eta^{*}\right) M(q) \mid M^{*}(p+q)\right\rangle=-g_{\mathrm{em}} g_{M^{*} M \gamma} \epsilon_{\mu \nu \rho \sigma} \eta^{* \mu} \varepsilon^{\nu} p^{\rho} q^{\sigma}
$$

can be readily deduced from the aforementioned electromagnetic decay form factor

$$
g_{M^{*} M \gamma}=\frac{2}{m_{V}+m_{P}} \mathcal{V}\left(p^{2}=0\right) .
$$

The general strategy of constructing the sum rules for the coupling $g_{M^{*} M \gamma}$ is to explore the vacuum-to-photon correlation function, following closely [8], defined with the two local interpolating currents for the vector and pseudoscalar heavy mesons

$$
\Pi_{\mu}(p, q)=\int d^{4} x e^{-i(p+q) \cdot x}\left\langle\gamma\left(p, \eta^{*}\right)\left|\mathrm{T}\left\{\bar{q}(x) \gamma_{\mu \perp} Q(x), \bar{Q}(0) \gamma_{5} q(0)\right\}\right| 0\right\rangle,
$$

where the perpendicular components of the Dirac $\gamma$ matrices are given by

$$
\gamma_{\mu \perp}=\gamma_{\mu}-\frac{\not h}{2} \bar{n}_{\mu}-\frac{\not h}{2} n_{\mu}, \quad p_{\mu}=\frac{n \cdot p}{2} \bar{n}_{\mu},
$$

with the light-cone vectors $n_{\mu}$ and $\bar{n}_{\mu}$ satisfying the constraints

$$
n \cdot \bar{n}=2, \quad n^{2}=\bar{n}^{2}=0 .
$$

To demonstrate the hard-collinear factorization formula for the correlation function (2.5) we will employ the following power counting scheme for the external momenta

$$
n \cdot p \sim \mathcal{O}\left(m_{Q}\right), \quad\left|(p+q)^{2}-m_{Q}^{2}\right| \sim \mathcal{O}\left(m_{Q}^{2}\right), \quad\left|q^{2}-m_{Q}^{2}\right| \sim \mathcal{O}\left(m_{Q}^{2}\right) .
$$

The leading power perturbative contribution to the vacuum-to-photon correlation function stems from the "point-like" photon component corresponding to the twist-one distribution amplitude [22] and can be further derived at tree level straightforwardly by evaluating the two triangle diagrams corresponding to the collinear photon coupling with the heavy-quark and light-quark electromagnetic currents as displayed in figure 1. 


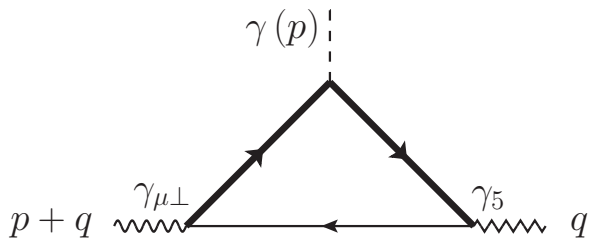

(a)

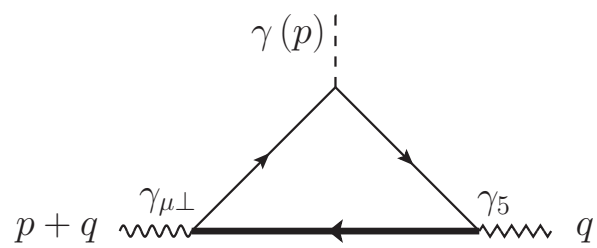

(b)

Figure 1. Diagrammatic representation of the "point-like" photon contribution to the vacuum-tophoton correlation function (2.5) at tree level.

The yielding contribution from the perturbative diagram in figure 1(a) is given by

$$
\begin{aligned}
\Pi_{\mu}^{1(\mathrm{a})}(p, q)= & -N_{c} e_{Q} g_{\mathrm{em}} \int \frac{d^{D} \ell}{(2 \pi)^{D}} \frac{1}{\left[(\ell-p-q)^{2}+i 0\right]\left[\ell^{2}-m_{Q}^{2}+i 0\right]\left[(\ell-p)^{2}-m_{Q}^{2}+i 0\right]} \\
& \operatorname{Tr}\left[\gamma_{\mu \perp}\left(\not \ell-\not p-\not q+m_{q}\right) \gamma_{5}\left(\not \ell-\not p+m_{Q}\right) \not \not^{*}\left(\not \ell+m_{Q}\right)\right],
\end{aligned}
$$

including the subleading power correction due to the light-quark mass at $\mathcal{O}\left(\Lambda_{\mathrm{QCD}} / m_{Q}\right)$. According to the power counting scheme (2.8) we can identify the leading power contribution of the following scalar loop integral

$$
I_{1}=\int \frac{d^{D} \ell}{(2 \pi)^{D}} \frac{1}{\left[(\ell-p-q)^{2}+i 0\right]\left[\ell^{2}-m_{Q}^{2}+i 0\right]\left[(\ell-p)^{2}-m_{Q}^{2}+i 0\right]}
$$

from the hard region in agreement with our expectation. It is then evident that the loopmomentum integration entering (2.9) is free of the ultraviolet and infrared divergences. Computing the Dirac algebra in the four-dimensional space leads to

$$
\begin{aligned}
\Pi_{\mu}^{1(\mathrm{a})}(p, q)= & -4 i N_{c} e_{Q} g_{\mathrm{em}} m_{Q} \int \frac{d^{D} \ell}{(2 \pi)^{D}} \frac{1}{\left[(\ell-p-q)^{2}+i 0\right]\left[\ell^{2}-m_{Q}^{2}+i 0\right]\left[(\ell-p)^{2}-m_{Q}^{2}+i 0\right]} \\
& \times\left[1-\left(1-r_{q}\right) \frac{\ell \cdot p}{p \cdot q}\right] \epsilon_{\mu p q \eta},
\end{aligned}
$$

with $r_{q}=m_{q} / m_{Q}$. Implementing the loop momentum integration subsequently yields

$$
\Pi_{\mu}^{1(\mathrm{a})}(p, q)=-\left(\frac{N_{c}}{4 \pi^{2}}\right) e_{Q} g_{\mathrm{em}} m_{Q} \epsilon_{\mu p q \eta} \int_{0}^{1} d y \int_{0}^{1} d t \frac{\bar{y}+y r_{q}}{m_{Q}^{2}-y \bar{t}\left(p+q^{2}\right)-y t q^{2}}
$$

where we have introduced the notations $\bar{y}=1-y, \bar{t}=1-t$ for brevity.

Along the same vein we can write down the perturbative contribution to the correlation function (2.5) due to the real photon radiation off the light quark

$$
\begin{aligned}
\Pi_{\mu}^{1(\mathrm{~b})}(p, q)= & -N_{c} e_{q} g_{\mathrm{em}} \int \frac{d^{D} \ell}{(2 \pi)^{D}} \frac{1}{\left[(\ell-p-q)^{2}-m_{Q}^{2}+i 0\right]\left[\ell^{2}+i 0\right]\left[(\ell-p)^{2}+i 0\right]} \\
& \operatorname{Tr}\left[\gamma_{\mu \perp}\left(\ell-\not p-\not 1+m_{Q}\right) \gamma_{5}\left(\ell-\not \ell+m_{q}\right) \not h^{*}\left(\ell+m_{q}\right)\right] \\
= & N_{c} e_{q} g_{\mathrm{em}} \int \frac{d^{D} \ell}{(2 \pi)^{D}} \frac{1}{\left[(\ell-p-q)^{2}-m_{Q}^{2}+i 0\right]\left[\ell^{2}+i 0\right]\left[(\ell-p)^{2}+i 0\right]} \\
& \left\{m_{Q} \operatorname{Tr}\left[\gamma_{\mu \perp} \gamma_{5} \not p \not 1^{*} \not \ell-m_{q} \operatorname{Tr}\left[\gamma_{\mu \perp} \gamma_{5} \not p \not h^{*}(\not \ell-\not 1)\right]\right\},\right.
\end{aligned}
$$


where we have explicitly separated the heavy-quark mass term from the light-quark mass correction to facilitate the power counting analysis for the diagram presented in figure 1(b). We are now in a position to determine all regions of the loop momentum yielding the leading power contributions to the two Feynman integrals in (2.13)

$$
\begin{aligned}
I_{2, A} & =\int \frac{d^{D} \ell}{(2 \pi)^{D}} \frac{\bar{n} \cdot \ell}{\left[(\ell-p-q)^{2}-m_{Q}^{2}+i 0\right]\left[\ell^{2}+i 0\right]\left[(\ell-p)^{2}+i 0\right]}, \\
I_{2, B} & =\int \frac{d^{D} \ell}{(2 \pi)^{D}} \frac{\bar{n} \cdot(\ell-q)}{\left[(\ell-p-q)^{2}-m_{Q}^{2}+i 0\right]\left[\ell^{2}+i 0\right]\left[(\ell-p)^{2}+i 0\right]} .
\end{aligned}
$$

Applying the default power counting scheme (2.8) it is straightforward to verify that $I_{2, A}$ receives the non-vanishing contribution only from the hard loop-momentum region and it therefore requires no ultraviolet and infrared subtractions in analogy to the scalar loop integral $I_{1}$. As a consequence, perturbative QCD factorization for the corresponding contribution to the correlation function (2.5) can indeed be established at leading power in $\Lambda_{\mathrm{QCD}} / m_{Q}$. By contrast, the remaining Feynman integral $I_{2, B}$ due to the light quark mass correction can be contributed from both the hard and collinear loop-momentum regions, where the typical scaling for a collinear momentum vector $\ell_{\mu}$ reads

$$
\ell_{\mu}=\left(n \cdot \ell, \bar{n} \cdot \ell, \ell_{\perp \mu}\right) \sim\left(1, \lambda^{2}, \lambda\right) m_{Q}, \quad \lambda=\Lambda_{\mathrm{QCD}} / m_{Q} .
$$

The emergence of the leading contribution to $I_{2, B}$ from the collinear momentum region implies that the nonperturbative photon distribution amplitudes must be introduced in the factorization formula to subtract the long-distance strong interaction effect from the perturbative QCD calculation of the correlation function (2.5). Since the $\mathrm{SU}(3)$-flavour symmetry breaking effect for the obtained light-cone matrix element $\left\langle\gamma\left(p, \eta^{*}\right)\left|\bar{q}(z) \gamma_{\mu \perp} \gamma_{5} q(0)\right| 0\right\rangle$ from the infrared subtraction program (see $[47,48]$ for discussions in the context of the vector meson distribution amplitudes) has not been investigated systematically at present, we will not take into account the light quark mass correction to the perturbative contribution shown in figure 1(b) further, which is apparently suppressed by one power of $\Lambda_{\mathrm{QCD}} / m_{Q}$ in the heavy quark expansion. Performing the loop momentum integration for the QCD amplitude $\Pi_{\mu}^{1(\mathrm{~b})}$ explicitly gives rise to

$$
\Pi_{\mu}^{1(\mathrm{~b})}(p, q) \supset-\left(\frac{N_{c}}{4 \pi^{2}}\right) e_{q} g_{\mathrm{em}} m_{Q} \epsilon_{\mu p q \eta} \int_{0}^{1} d y \int_{0}^{1} d t \frac{y}{m_{Q}^{2}-y \bar{t}\left(p+q^{2}\right)-y t q^{2}} .
$$

Adding up the two different pieces of perturbative contributions displayed in (2.12) and (2.17) yields the tree-level factorization formula for the "point-like" photon contribution

$$
\Pi_{\mu}^{(\mathrm{per})}(p, q) \supset-\left(\frac{N_{c}}{4 \pi^{2}}\right) g_{\mathrm{em}} m_{Q} \epsilon_{\mu p q \eta} \int_{0}^{1} d y \int_{0}^{1} d t \frac{e_{Q}\left(\bar{y}+y r_{q}\right)+e_{q} y}{m_{Q}^{2}-y \bar{t}\left(p+q^{2}\right)-y t q^{2}},
$$

which can be expressed in terms of the following dispersion integral

$$
\Pi_{\mu}^{(\mathrm{per})}(p, q) \supset-\left(\frac{N_{c}}{4 \pi^{2}}\right) g_{\mathrm{em}} m_{Q} \epsilon_{\mu p q \eta} \int d s_{1} \int d s_{2} \frac{\rho^{(\mathrm{per})}\left(s_{1}, s_{2}\right)}{\left[s_{1}-(p+q)^{2}-i 0\right]\left[s_{2}-q^{2}-i 0\right]},
$$


with the QCD spectral density at LO given by

$$
\begin{aligned}
\rho^{(\mathrm{per})}\left(s_{1}, s_{2}\right)= & -\delta\left(s_{1}-s_{2}\right)\left\{e_{Q}\left[\left(1-r_{q}\right)\left(1-\frac{m_{Q}^{2}}{s_{2}}\right)+\ln \left(\frac{m_{Q}^{2}}{s_{2}}\right)\right]-e_{q}\left(1-\frac{m_{Q}^{2}}{s_{2}}\right)\right\} \\
& \times \theta\left(s_{2}-m_{Q}^{2}\right)+\mathcal{O}\left(\alpha_{s}\right) .
\end{aligned}
$$

Taking advantage of the standard definitions for the heavy meson decay constants

$$
\left\langle 0\left|\bar{Q} \gamma_{5} q\right| M(q)\right\rangle=-i \frac{f_{P} m_{P}^{2}}{m_{Q}+m_{q}}, \quad\left\langle M^{*}(p+q)\left|\bar{q} \gamma_{\mu \perp} Q\right| 0\right\rangle=-i f_{V} m_{V} \varepsilon_{\mu \perp}^{*}
$$

and for the coupling $g_{M^{*} M \gamma}$ as introduced in (2.3), we can readily derive the hadronic dispersion relation for the vacuum-to-photon correlation function (2.5)

$$
\begin{aligned}
\Pi_{\mu}(p, q)= & -\epsilon_{\mu p q \eta}\left\{\frac{g_{\mathrm{em}} f_{P} f_{V} m_{V} g_{M^{*} M \gamma}}{\left[m_{V}^{2}-(p+q)^{2}-i 0\right]\left[m_{P}^{2}-q^{2}-i 0\right]} \frac{m_{P}^{2}}{m_{Q}+m_{q}}\right. \\
& \left.+\int d s_{1} \int d s_{2} \frac{\rho^{(\mathrm{had})}\left(s_{1}, s_{2}\right)}{\left[s_{1}-(p+q)^{2}-i 0\right]\left[s_{2}-q^{2}-i 0\right]}\right\} .
\end{aligned}
$$

Matching the perturbative QCD factorization formula (2.19) of the "point-like" photon contribution with the corresponding hadronic representation (2.22) and performing the double Borel transformation with respect to the variables $(p+q)^{2} \rightarrow M_{1}^{2}$ and $q^{2} \rightarrow M_{2}^{2}$, we derive the sum rules for the coupling constant $g_{M^{*} M \gamma}$ of our interest including the light quark mass correction

$$
\begin{aligned}
& f_{P} f_{V} \mu_{P} m_{V} g_{M^{*} M \gamma}^{(\mathrm{per})} \exp \left[-\left(\frac{m_{V}^{2}}{M_{1}^{2}}+\frac{m_{P}^{2}}{M_{2}^{2}}\right)\right] \\
& =\left(\frac{N_{c}}{4 \pi^{2}}\right) m_{Q} \iint_{\Sigma} d s_{1} d s_{2} \exp \left[-\left(\frac{s_{1}}{M_{1}^{2}}+\frac{s_{2}}{M_{2}^{2}}\right)\right] \rho^{(\mathrm{per})}\left(s_{1}, s_{2}\right),
\end{aligned}
$$

where $\mu_{P}=m_{P}^{2} /\left(m_{Q}+m_{q}\right)$ and the appearance of the integration boundary $\Sigma$ in the $\left(s_{1}, s_{2}\right)$ plane arises from subtracting the continuum contributions with the parton-hadron duality ansatz. Since the Borel parameters $M_{1}^{2}$ and $M_{2}^{2}$ are expected to be of similar size numerically, we will simplify the generic expression of the LCSR (2.23) by making the approximation $M_{1}^{2}=M_{2}^{2} \equiv M^{2}$ in what follows. Employing the triangle duality region $s_{1}+s_{2} \leq 2 s_{0}$ as suggested in [43,44] and introducing further the Jacobi transformation

$$
s_{1}+s_{2}=2 s, \quad v=\frac{s_{2}}{s_{1}+s_{2}},
$$

we obtain the tree-level LCSR for the "point-like" photon contribution

$$
\begin{aligned}
f_{P} f_{V} \mu_{P} m_{V} g_{M^{*} M \gamma}^{(\mathrm{per})}= & -\left(\frac{N_{c}}{4 \pi^{2}}\right) m_{Q} \int_{m_{Q}^{2}}^{s_{0}} d s \exp \left[-\frac{2 s-m_{V}^{2}-m_{P}^{2}}{M^{2}}\right] \\
& \times\left\{e_{Q}\left[\left(1-r_{q}\right)\left(1-\frac{m_{Q}^{2}}{s}\right)+\ln \frac{m_{Q}^{2}}{s}\right]-e_{q}\left(1-\frac{m_{Q}^{2}}{s}\right)\right\}
\end{aligned}
$$


which can be reduced further by integrating over the variable $s$ analytically

$$
\begin{aligned}
f_{P} f_{V} \mu_{P} m_{V} g_{M^{*} M \gamma}^{(\text {per })}= & \left(\frac{N_{c}}{8 \pi^{2}}\right) m_{Q}^{3} \exp \left[\frac{m_{V}^{2}+m_{P}^{2}}{M^{2}}\right] \\
& \times\left\{\left[\operatorname{Ei}\left(-\frac{2 s_{0}}{M^{2}}\right)-\operatorname{Ei}\left(-\frac{2 m_{Q}^{2}}{M^{2}}\right)\right]\left[e_{Q}\left(2\left(1-r_{q}\right)+\frac{M^{2}}{m_{Q}^{2}}\right)-2 e_{q}\right]\right. \\
& +\frac{M^{2}}{m_{Q}^{2}}\left[\exp \left(-\frac{2 s_{0}}{M^{2}}\right)-\exp \left(-\frac{2 m_{Q}^{2}}{M^{2}}\right)\right]\left[e_{Q}\left(1-r_{q}\right)-e_{q}\right] \\
& \left.-\frac{M^{2}}{m_{Q}^{2}} \exp \left(-\frac{2 s_{0}}{M^{2}}\right)\left[e_{Q} \ln \frac{s_{0}}{m_{Q}^{2}}\right]\right\}
\end{aligned}
$$

with the exponential integral $\operatorname{Ei}(x)$ defined as

$$
\operatorname{Ei}(x)=-\int_{-x}^{\infty} d t \frac{e^{-t}}{t}, \quad(x \neq 0)
$$

Several remarks on the obtained sum rules (2.26) for the perturbative contribution to the coupling $g_{M^{*} M \gamma}$ with the light quark mass correction are in order.

- It would be straightforward to explore the heavy-quark mass dependence of all separate terms entering the obtained sum rules (2.26) based upon the power counting scheme $[49,50]$

$$
m_{V} \sim m_{P} \sim m_{Q}+\Lambda_{\mathrm{QCD}}, \quad s_{0} \sim\left(m_{Q}+\omega_{0}\right)^{2}, \quad f_{P} \sim f_{V} \sim \Lambda_{\mathrm{QCD}}^{3 / 2} / m_{Q}^{1 / 2},
$$

where the $m_{Q}$-independent parameter $\omega_{0}$ is of order $1 \mathrm{GeV}$. Expanding (2.26) in terms of the electrical charges of the heavy and light quarks

$$
g_{M^{*} M \gamma}^{(\mathrm{per})}=e_{Q} g_{M^{*} M \gamma}^{(\mathrm{per}, \mathrm{I})}+e_{Q} r_{q} g_{M^{*} M \gamma}^{(\mathrm{per}, \mathrm{II})}+e_{q} g_{M^{*} M \gamma}^{(\mathrm{per}, \mathrm{III})},
$$

we can immediately establish the asymptotic scaling laws

$$
g_{M^{*} M \gamma}^{(\mathrm{per}, \mathrm{I})} \sim \frac{1}{m_{Q}}\left(\frac{\omega_{0}}{\Lambda_{\mathrm{QCD}}}\right)^{3}, \quad g_{M^{*} M \gamma}^{(\mathrm{per}, \mathrm{II})} \sim g_{M^{*} M \gamma}^{(\mathrm{per}, \mathrm{III})} \sim \frac{1}{\Lambda_{\mathrm{QCD}}}\left(\frac{\omega_{0}}{\Lambda_{\mathrm{QCD}}}\right)^{2},
$$

in agreement with the $\mathrm{HH} \chi \mathrm{PT}$ predictions [24-26].

- The substantial cancellation of two different pieces from the photon radiation off the charm and light quarks can be naturally expected for the magnetic couplings $D_{(s)}^{*+} D_{(s)}^{+} \gamma$. Consequently, it remains interesting to investigate whether the higherorder QCD corrections to the "point-like" photon contributions (see [51] for discussions in the context of $\left.J / \psi \rightarrow \eta_{c} \gamma\right)$ could alleviate such numerical cancellation. 


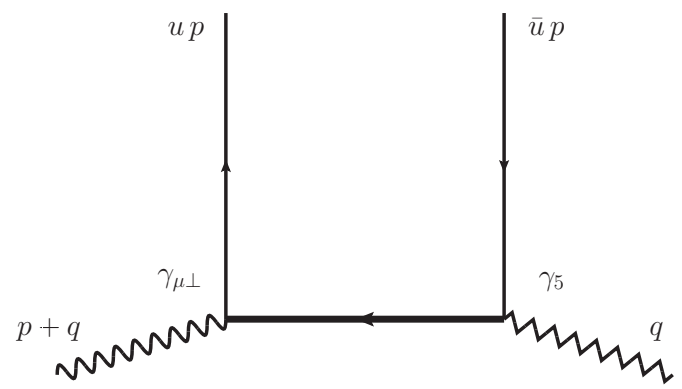

Figure 2. Diagrammatic representation of the four-point QCD matrix element $F_{\mu}(p, q, u)$ defined by (3.1) at tree level.

\section{The twist-two LCSR for the resolved photon effect}

We will proceed to compute the twist-two hadronic photon corrections to the radiative heavy meson decay form factors at NLL by employing the LCSR approach. To this end, we need to establish the corresponding hard-collinear factorization formula for the vacuumto-photon correlation function $(2.5)$ at $\mathcal{O}\left(\alpha_{s}\right)$ with the evanescent operator approach and then implement the RG resummation for enhanced logarithms of the hard-to-collinear scale ratio with the two-loop evolution equation of the twist-two photon LCDA.

\subsection{Hard-collinear factorization at LO in QCD}

Following the standard strategy, the perturbative QCD factorization formula for the correlation function (2.5) can be established by inspecting the partonic matrix element

$$
F_{\mu}(p, q, u)=\int d^{4} x e^{-i(p+q) \cdot x}\left\langle q(u p) \bar{q}(\bar{u} p)\left|\mathrm{T}\left\{\bar{q}(x) \gamma_{\mu \perp} Q(x), \bar{Q}(0) \gamma_{5} q(0)\right\}\right| 0\right\rangle,
$$

where the dimensionless parameter $u \in[0,1]$ corresponds to the momentum fraction carried by the collinear quark and $\bar{u} \equiv 1-u$. Evaluating the leading-order Feynman diagram depicted in figure 2 gives rise to the tree-level QCD amplitude

$$
\begin{aligned}
F_{\mu}^{(0)}(p, q, u) & =-\frac{i}{2} \frac{\bar{n} \cdot q}{\bar{u}(p+q)^{2}+u q^{2}-m_{Q}^{2}+i 0} \bar{q}(u p) \gamma_{\mu \perp} \not h \gamma_{5} q(\bar{u} p) \\
& =-\frac{i}{2} \frac{\bar{n} \cdot q}{\bar{u}^{\prime}(p+q)^{2}+u^{\prime} q^{2}-m_{Q}^{2}+i 0} *\left\langle O_{1, \mu}\left(u, u^{\prime}\right)\right\rangle^{(0)}
\end{aligned}
$$

where the asterisk represents the convolution integral over the variable $u^{\prime}$ and the quark matrix element $\left\langle O_{1, \mu}\left(u, u^{\prime}\right)\right\rangle$ of the two-body light-ray operator is given by

$$
\left\langle O_{1, \mu}\left(u, u^{\prime}\right)\right\rangle=\left\langle q(u p) \bar{q}(\bar{u} p)\left|\hat{O}_{1, \mu}\left(u^{\prime}\right)\right| 0\right\rangle=\bar{\xi}(u p) \gamma_{\mu \perp} \not h \gamma_{5} \xi(\bar{u} p) \delta\left(u-u^{\prime}\right)+\mathcal{O}\left(\alpha_{s}\right) .
$$

The collinear operator with a given Dirac structure $\Gamma_{j}$ in momentum space is defined as [52]

$$
\hat{O}_{j, \mu}\left(u^{\prime}\right)=\frac{n \cdot p}{2 \pi} \int d \tau e^{-i u^{\prime} n \cdot p \tau}\left(\bar{\xi} W_{c}\right)(\tau n) \Gamma_{j}\left(W_{c}^{\dagger} \xi\right)(0), \quad \Gamma_{1}=\gamma_{\mu \perp} \not h \gamma_{5},
$$


where the collinear gauge invariance is satisfied by introducing the light-like Wilson line

$$
W_{c}(x)=\mathrm{P}\left\{\exp \left[i g_{s} \int_{-\infty}^{0} d s n \cdot A_{c}(x+s n)\right]\right\} .
$$

Implementing the infrared subtraction for the renormalized matrix element $F_{\mu}(p, q, u)$ can be further achieved with the aid of the collinear operator basis

$$
\hat{O}_{1, \mu}=\hat{O}_{2, \mu}+\hat{O}_{E, \mu}, \quad \Gamma_{2}=\frac{n^{\nu}}{2} \epsilon_{\mu \nu \rho \tau} \sigma^{\rho \tau}, \quad \Gamma_{E}=\gamma_{\mu \perp} \not h \gamma_{5}-\frac{n^{\nu}}{2} \epsilon_{\mu \nu \rho \tau} \sigma^{\rho \tau},
$$

where the evanescent operator $\hat{O}_{E, \mu}$ vanishes in the four-dimensional space-time. Taking advantage of the perturbative matching relation

$$
F_{\mu}(p, q, u)=\sum_{i=2, E} H_{i}\left((p+q)^{2}, q^{2}, u^{\prime}\right) *\left\langle O_{i, \mu}\left(u, u^{\prime}\right)\right\rangle
$$

we can readily obtain the short-distance coefficient functions at tree level

$$
H_{2}^{(0)}=H_{E}^{(0)}=-\frac{i}{2} \frac{\bar{n} \cdot q}{\bar{u}^{\prime}(p+q)^{2}+u^{\prime} q^{2}-m_{Q}^{2}+i 0} .
$$

It is then straightforward to derive the hard-collinear factorization formula for the vacuumto-photon correlation function (2.5) at leading power in $\Lambda_{\mathrm{QCD}} / m_{Q}$

$$
\Pi_{\mu, \mathrm{LO}}^{\mathrm{tw} 2}(p, q)=-g_{\mathrm{em}} e_{q} \chi(\mu)\langle\bar{q} q\rangle(\mu) \epsilon_{\mu p q \eta^{*}} \int_{0}^{1} d u \frac{\phi_{\gamma}(u, \mu)}{\bar{u}(p+q)^{2}+u q^{2}-m_{Q}^{2}+i 0},
$$

by employing the definition of the twist-two photon distribution amplitude

$$
\begin{aligned}
& \left\langle\gamma\left(p, \eta^{*}\right)\left|\left(\bar{\xi} W_{c}\right)(\tau n) \sigma_{\alpha \beta}\left(W_{c}^{\dagger} \xi\right)(0)\right| 0\right\rangle \\
& =-i g_{\mathrm{em}} e_{q} \chi(\mu)\langle\bar{q} q\rangle(\mu)\left(p_{\beta} \eta_{\alpha}^{*}-p_{\alpha} \eta_{\beta}^{*}\right) \int_{0}^{1} d u e^{i u n \cdot p \tau} \phi_{\gamma}(u, \mu) .
\end{aligned}
$$

We proceed to write down the dispersion representation of the factorization formula (3.9)

$$
\begin{aligned}
\Pi_{\mu, \mathrm{LO}}^{\mathrm{tw} 2}(p, q)= & -g_{\mathrm{em}} e_{q} \chi(\mu)\langle\bar{q} q\rangle(\mu) \epsilon_{\mu p q \eta^{*}} \\
& \times \int_{m_{Q}^{2}}^{\infty} d s_{1} \int_{m_{Q}^{2}}^{\infty} d s_{2} \frac{\rho_{0}^{\mathrm{tw} 2}\left(s_{1}, s_{2}\right)}{\left[s_{1}-(p+q)^{2}-i 0\right]\left[s_{2}-q^{2}-i 0\right]}
\end{aligned}
$$

where the LO double spectral density [44]

$$
\rho_{0}^{\mathrm{tw} 2}\left(s_{1}, s_{2}\right)=\sum_{k} \frac{(-1)^{k+1} a_{k}(\mu)}{\Gamma(k+1)}\left(s_{1}-m_{Q}^{2}\right)^{k} \delta^{(k)}\left(s_{1}-s_{2}\right),
$$

is obtained by expanding the twist-two photon LCDA in terms of the polynomials in $u$

$$
\phi_{\gamma}(u, \mu)=\sum_{k} a_{k}(\mu) u^{k} .
$$




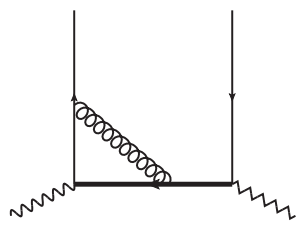

(a)

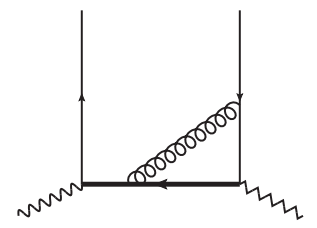

(b)

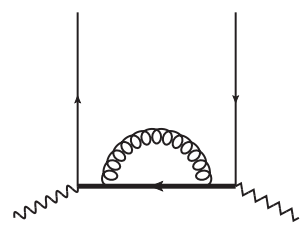

(c)

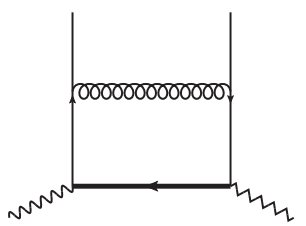

(d)

Figure 3. Diagrammatic representation of the four-point QCD matrix element $F_{\mu}(p, q, u)$ defined by (3.1) at one loop.

The resulting LCSR for the resolved photon contribution to the magnetic coupling $g_{M^{*} M \gamma}$ is given by

$$
f_{P} f_{V} \mu_{P} m_{V} g_{M^{*} M \gamma}^{(\mathrm{tw} 2, \mathrm{LO})}=-e_{q} \chi(\mu)\langle\bar{q} q\rangle(\mu) \phi_{\gamma}\left(\frac{1}{2}, \mu\right) \int_{m_{Q}^{2}}^{s_{0}} d s \exp \left[\frac{m_{V}^{2}+m_{P}^{2}-2 s}{M^{2}}\right] .
$$

Applying the default power counting scheme displayed in (2.28) we find

$$
g_{M^{*} M \gamma}^{(\mathrm{tw} 2, \mathrm{LO})} \sim \frac{1}{\Lambda_{\mathrm{QCD}}}\left(\frac{\omega_{0}}{\Lambda_{\mathrm{QCD}}}\right),
$$

which is suppressed by a factor of $\Lambda_{\mathrm{QCD}} / \omega_{0}$ compared with the "point-like" photon effect.

\subsection{Hard-collinear factorization at NLO in QCD}

We are now in a position to demonstrate the hard-collinear factorization formula for the vacuum-to-photon correlation function (2.5) at NLO in QCD. The determination of the corresponding perturbative matching coefficient can be achieved by evaluating the one-loop diagrams displayed in figure 3 with the aid of (3.7). Evaluating the QCD correction to the vector-meson vertex diagram shown in figure 3 (a) leads to

$$
\begin{aligned}
F_{\mu}^{(1, a)}= & \frac{g_{s}^{2} C_{F}}{\bar{u}(p+q)^{2}+u q^{2}-m_{Q}^{2}} \int \frac{d^{D} \ell}{(2 \pi)^{D}} \frac{1}{\left[(\ell+u p)^{2}+i 0\right]\left[(\ell-\bar{u} p-q)^{2}-m_{Q}^{2}+i 0\right]\left[\ell^{2}+i 0\right]} \\
& \bar{q}(u p) \gamma_{\nu}(u \not p+\not) \gamma_{\mu \perp}\left(\not \ell-\bar{u} \not p-\not q+m_{Q}\right) \gamma^{\nu}\left(-\bar{u} \not p-\not q+m_{Q}\right) \gamma_{5} q(\bar{u} p) .
\end{aligned}
$$

Applying the power counting scheme for the external momenta presented in (2.8), we can identify the leading power contributions of the scalar Feynman integral

$$
I_{a}=\int \frac{d^{D} \ell}{(2 \pi)^{D}} \frac{1}{\left[(\ell+u p)^{2}+i 0\right]\left[(\ell-\bar{u} p-q)^{2}-m_{Q}^{2}+i 0\right]\left[\ell^{2}+i 0\right]}
$$

from the hard and collinear regions. Employing the strategy of regions constructed in $[41,42]$, the collinear region integral at leading power in the heavy quark expansion can be reduced as

$$
I_{a}^{(c)}=-\int \frac{d^{D} \ell}{(2 \pi)^{D}} \frac{1}{\left[n \cdot(\ell+u p) \bar{n} \cdot \ell+\ell_{\perp}^{2}+i 0\right]\left[n \cdot(\ell-\bar{u} p-q) \bar{n} \cdot q+m_{Q}^{2}-i 0\right]\left[\ell^{2}+i 0\right]}
$$


which results in a vanishing contribution with the dimensional regularization scheme and will be also cancelled out by the corresponding collinear subtraction term with an arbitrary regularization scheme. Taking advantage of the expressions for the hard-region integrals collected in appendix A as well as the naive dimensional regularization (NDR) scheme for the Dirac matrix $\gamma_{5}$, the one-loop correction to the vector-meson vertex diagram can be computed as

$$
\begin{aligned}
F_{\mu}^{(1, a)}= & \frac{\alpha_{s} C_{F}}{4 \pi}\left\{\left[\frac{1}{\epsilon}+\ln \frac{\mu^{2}}{m_{Q}^{2}}-\ln \left[\left(1-r_{1}\right)\left(1-r_{2}\right)\right]-\frac{r_{1}-2 r_{2}+1}{2 r_{1}\left(1-r_{2}\right)}+\frac{3}{2}\right]\right. \\
& \times\left[\frac{2\left(1-r_{2}\right)}{r_{2}-r_{1}} \ln \frac{1-r_{1}}{1-r_{2}}-1\right]+\frac{2\left(1-r_{2}\right)}{r_{1}-r_{2}}\left[\operatorname{Li}_{2}\left(r_{1}\right)-\operatorname{Li}_{2}\left(r_{2}\right)\right] \\
& \left.-\left(\frac{1-2 r_{2}}{r_{1} r_{2}}+1\right) \ln \left(1-r_{2}\right)-\frac{r_{1}-2 r_{2}+1}{2 r_{1}\left(1-r_{2}\right)}-\frac{5}{2}\right\} F_{\mu}^{(0)},
\end{aligned}
$$

where we have introduced the following dimensionless variables

$$
r_{1} \equiv(\bar{u} p+q)^{2} / m_{Q}^{2}, \quad r_{2} \equiv(p+q)^{2} / m_{Q}^{2} .
$$

Along the same vein, we can write down the one-loop amplitude for the QCD correction to the pseudoscalar-meson vertex diagram displayed in figure 3 (b)

$$
\begin{aligned}
F_{\mu}^{(1, b)}= & \frac{g_{s}^{2} C_{F}}{\bar{u}(p+q)^{2}+u q^{2}-m_{Q}^{2}} \int \frac{d^{D} \ell}{(2 \pi)^{D}} \frac{1}{\left[(\ell-\bar{u} p)^{2}+i 0\right]\left[(\ell-\bar{u} p-q)^{2}-m_{Q}^{2}+i 0\right]\left[\ell^{2}+i 0\right]} \\
& \bar{q}(u p) \gamma_{\mu \perp}\left(-\bar{u} \not p-\not q+m_{Q}\right) \gamma_{\nu}\left(\not \ell-\bar{u} \not p-\not q+m_{Q}\right) \gamma_{5}(\not \ell-u \not p) \gamma^{\nu} q(\bar{u} p) . \quad(3.21)
\end{aligned}
$$

Apparently, only the hard and collinear loop-momentum regions can generate the leading power contributions to $F_{\mu}^{(1, b)}$ with the power counting scheme (2.8). Implementing the Dirac algebra reduction with the equation of motion for the quark field and performing the loop-momentum integration with the formulae displayed in appendix A, we obtain

$$
\begin{aligned}
F_{\mu}^{(1, b)}= & \frac{\alpha_{s} C_{F}}{2 \pi}\left\{\left[\frac{1}{\epsilon}+\ln \frac{\mu^{2}}{m_{Q}^{2}}-\ln \left[\left(1-r_{1}\right)\left(1-r_{3}\right)\right]-\frac{r_{3}-2 r_{1}+1}{r_{1}\left(1-r_{3}\right)}\right]\right. \\
& \times\left[\frac{1-r_{3}}{r_{3}-r_{1}} \ln \frac{1-r_{1}}{1-r_{3}}+1\right]+\frac{1-r_{3}}{r_{1}-r_{3}}\left[\operatorname{Li}_{2}\left(r_{1}\right)-\mathrm{Li}_{2}\left(r_{3}\right)\right] \\
& \left.+\left(\frac{r_{3}+1}{r_{1} r_{3}}+1\right) \ln \left(1-r_{3}\right)-\frac{r_{3}-2 r_{1}+1}{r_{1}\left(1-r_{3}\right)}+1\right\} F_{\mu}^{(0)},
\end{aligned}
$$

where we have defined the convention $r_{3} \equiv q^{2} / m_{Q}^{2}$ for brevity.

The self-energy correction to the heavy-quark propagator shown in figure 3 (c) is evidently free of the collinear divergence and can be readily computed as follows

$$
\begin{aligned}
F_{\mu}^{(1, c)}= & \frac{g_{s}^{2} C_{F}}{\bar{u}(p+q)^{2}+u q^{2}-m_{Q}^{2}} \int \frac{d^{D} \ell}{(2 \pi)^{D}} \frac{1}{\left[(\ell-\bar{u} p-q)^{2}-m_{Q}^{2}+i 0\right]\left[\ell^{2}+i 0\right]} \\
& \bar{q}(u p) \gamma_{\mu \perp}\left(-\bar{u} \not p-\not q+m_{Q}\right) \gamma_{\nu}\left(\not \ell-\bar{u} \not p-\not q+m_{Q}\right) \gamma^{\nu}\left(-\bar{u} \not p-\not q+m_{Q}\right) \gamma_{5} q(\bar{u} p), \\
= & -\frac{\alpha_{s} C_{F}}{4 \pi}\left\{\frac{7-r_{1}}{1-r_{1}}\left[\frac{1}{\epsilon}+\ln \frac{\mu^{2}}{m_{Q}^{2}}-\ln \left(1-r_{1}\right)+1\right]\right. \\
& \left.-\frac{1}{1-r_{1}}\left[\frac{1-7 r_{1}}{r_{1}^{2}} \ln \left(1-r_{1}\right)+\frac{1}{r_{1}}-3\right]\right\} F_{\mu}^{(0)},
\end{aligned}
$$

by employing the results of the one-loop Feynman integrals collected in appendix A. 
The one-loop QCD amplitude from the box diagram displayed in figure $3(\mathrm{~d})$ is given by

$$
\begin{aligned}
F_{\mu}^{(1, d)}= & g_{s}^{2} C_{F} \int \frac{d^{D} \ell}{(2 \pi)^{D}} \frac{1}{\left[(\ell-\bar{u} p)^{2}+i 0\right]\left[(\ell+u p)^{2}+i 0\right]\left[(\ell-\bar{u} p-q)^{2}-m_{Q}^{2}+i 0\right]\left[\ell^{2}+i 0\right]} \\
& \bar{q}(u p) \gamma_{\nu}(\ell+u \not p) \gamma_{\mu \perp}\left(\not \ell-\bar{u} \not p-\not q+m_{Q}\right) \gamma_{5}(\not \ell-\bar{u} \not p) \gamma^{\nu} q(\bar{u} p),
\end{aligned}
$$

which can be further reduced with the NDR scheme of $\gamma_{5}$

$$
\begin{aligned}
F_{\mu}^{(1, d)}= & i g_{s}^{2} C_{F} \int \frac{d^{D} \ell}{(2 \pi)^{D}} \frac{m_{Q}^{2}\left(1-r_{1}\right)}{\left[(\ell-\bar{u} p)^{2}+i 0\right]\left[(\ell+u p)^{2}+i 0\right]\left[(\ell-\bar{u} p-q)^{2}-m_{Q}^{2}+i 0\right]\left[\ell^{2}+i 0\right]} \\
& (D-4)\left\{\frac{D-4}{D-2} \ell_{\perp}^{2}+\frac{\bar{n} \cdot \ell}{\bar{n} \cdot q}\left[\ell^{2}-\bar{n} \cdot \ell n \cdot(\bar{u} p+q)\right]\right\} F_{\mu}^{(0)} .
\end{aligned}
$$

Evaluating the tensor four-point integrals with the dimensional regularization scheme implies that the obtained amplitude $F_{\mu}^{(1, d)}$ only contributes at $\mathcal{O}(\epsilon)$ and therefore vanishes at $D=4$, in agreement with the analogous observation for the hadronic photon corrections to the radiative leptonic $B$-meson decays [8].

Adding up different pieces together we can derive the one-loop contribution to the QCD matrix element $F_{\mu}$ as follows

$$
F_{\mu}^{(1)}(p, q, u)=\sum_{i=2, E} T_{i}^{(1)}\left((p+q)^{2}, q^{2}, u^{\prime}\right) *\left\langle O_{i, \mu}\left(u, u^{\prime}\right)\right\rangle^{(0)}
$$

where the explicit expressions of the hard amplitudes $T_{i}^{(1)}$ with the NDR scheme of $\gamma_{5}$ read

$$
\begin{aligned}
& T_{i}^{(1)}\left((p+q)^{2}, q^{2}, u^{\prime}\right) \\
&= \frac{\alpha_{s} C_{F}}{4 \pi}\left\{(-2)\left[\frac{1-r_{2}}{r_{1}-r_{2}} \ln \frac{1-r_{1}}{1-r_{2}}+\frac{1-r_{3}}{r_{1}-r_{3}} \ln \frac{1-r_{1}}{1-r_{3}}+\frac{3}{1-r_{1}}\right]\left(\frac{1}{\epsilon}+\ln \frac{\mu^{2}}{m_{Q}^{2}}\right)\right. \\
&+2\left[\left(\frac{1-r_{2}}{r_{1}-r_{2}}+\frac{1-r_{3}}{r_{1}-r_{3}}\right) \operatorname{Li}_{2}\left(r_{1}\right)-\frac{1-r_{2}}{r_{1}-r_{2}} \operatorname{Li}_{2}\left(r_{2}\right)-\frac{1-r_{3}}{r_{1}-r_{3}} \operatorname{Li}_{2}\left(r_{3}\right)\right] \\
&+2\left[\left(\frac{1-r_{2}}{r_{1}-r_{2}}+\frac{1-r_{3}}{r_{1}-r_{3}}\right) \ln ^{2}\left(1-r_{1}\right)-\frac{1-r_{2}}{r_{1}-r_{2}} \ln ^{2}\left(1-r_{2}\right)-\frac{1-r_{3}}{r_{1}-r_{3}} \ln ^{2}\left(1-r_{3}\right)\right] \\
&+\left[\frac{1-r_{1}}{r_{1}}\left(\frac{1-r_{1}-2 r_{2}}{r_{1}-r_{2}}-\frac{2\left(1-r_{1}+r_{3}\right)}{r_{1}-r_{3}}\right)+\frac{1-6 r_{1}}{r_{1}^{2}}+1\right] \ln \left(1-r_{1}\right) \\
&\left.+\frac{1-9 r_{1}}{r_{1}\left(1-r_{1}\right)}-\frac{\left(1-r_{2}\right)\left(1-3 r_{2}\right)}{r_{2}\left(r_{1}-r_{2}\right)} \ln \left(1-r_{2}\right)+\frac{2\left(1-r_{3}\right)}{r_{3}\left(r_{1}-r_{3}\right)} \ln \left(1-r_{3}\right)-3\right\} H_{i}^{(0)},
\end{aligned}
$$

with the variable $u$ in the definition of $r_{1}$ replaced by $u^{\prime}$. Expanding all the quantities in the operator matching relation (3.7) at the one-loop accuracy yields

$$
\begin{aligned}
\sum_{i} T_{i}^{(1)}\left((p+q)^{2}, q^{2}, u^{\prime}\right) *\left\langle O_{i, \mu}\left(u, u^{\prime}\right)\right\rangle^{(0)}= & \sum_{i}\left[H_{i}^{(1)}\left((p+q)^{2}, q^{2}, u^{\prime}, \mu\right) *\left\langle O_{i, \mu}\left(u, u^{\prime}\right)\right\rangle^{(0)}\right. \\
& \left.+H_{i}^{(0)}\left((p+q)^{2}, q^{2}, u^{\prime}\right) *\left\langle O_{i, \mu}\left(u, u^{\prime}, \mu\right)\right\rangle^{(1)}\right] .(3
\end{aligned}
$$

The ultraviolet renormalized collinear matrix elements $\left\langle O_{i, \mu}\right\rangle^{(1)}$ are given by

$$
\left\langle O_{i, \mu}\right\rangle^{(1)}=\left[M_{i j}^{(1), R}+Z_{i j}^{(1)}\right]\left\langle O_{j, \mu}\right\rangle^{(0)},
$$


where $M_{i j}^{(1), R}$ and $Z_{i j}^{(1)}$ represent the bare matrix elements computed from the infrared regularization scheme $R$ and the renormalization factors for subtracting the ultraviolet divergences at one loop, respectively. Following the prescriptions constructed in [53], the one-loop hard matching coefficient $H_{2}^{(1)}$ of the physical operator $\hat{O}_{2, \mu}$ can be determined as

$$
H_{2}^{(1)}=T_{2}^{(1)}-H_{2}^{(0)} * Z_{22}^{(1)}+H_{E}^{(0)} * M_{E 2}^{(1), \text { off }},
$$

where the infrared subtraction term $H_{E}^{(0)} * M_{E 2}^{(1) \text {, off }}$ arises from the mixing of $\hat{O}_{E, \mu}$ into $\hat{O}_{2, \mu}$. Computing the one-loop matrix element of the evanescent operator explicitly leads to $[8]$

$$
H_{E}^{(0)} * M_{E 2}^{(1), \text { off }}=0
$$

from which we can immediately write down the desired short-distance matching coefficient

$$
H_{2}^{(1)}=T_{2}^{(1), \text { reg }}
$$

It is apparent that $T_{2}^{(1) \text {, reg }}$ corresponds to the regularized terms of the NLO QCD matrix element $T_{2}^{(1)}$ displayed in (3.27).

The one-loop hard-collinear factorization formula for the vacuum-to-photon correlation function at the twist-two accuracy is then given by

$$
\begin{aligned}
\Pi_{\mu, \mathrm{NLO}}^{\mathrm{tw} 2}(p, q)= & -g_{\mathrm{em}} e_{q} \chi(\mu)\langle\bar{q} q\rangle(\mu) \epsilon_{\mu p q \eta^{*}} \int_{0}^{1} d u \frac{\phi_{\gamma}(u, \mu)}{\bar{u}(p+q)^{2}+u q^{2}-m_{Q}^{2}+i 0} \\
& {\left[1+\frac{H_{2}^{(1)}\left((p+q)^{2}, q^{2}, u, \mu\right)}{H_{2}^{(0)}\left((p+q)^{2}, q^{2}, u\right)}\right]+\mathcal{O}\left(\alpha_{s}^{2}\right) . }
\end{aligned}
$$

In order to demonstrate the factorization-scale independence of the obtained expression for the correlation function (2.5), we need to distinguish the QCD renormalization scale $\nu$ for the pseudoscalar interpolating current with the hard-collinear factorization scale $\mu$. Such distinction can be explicitly achieved by employing the following decomposition

$$
H_{2}^{(1)}\left((p+q)^{2}, q^{2}, u, \mu, \nu\right)=H_{2}^{(1)}\left((p+q)^{2}, q^{2}, u, \mu\right)+\delta H_{2}^{(1)}\left((p+q)^{2}, q^{2}, u, \mu, \nu\right),
$$

where the second term on the right-hand side $\delta H_{2}^{(1)}$ satisfies the RG evolution equation

$$
\frac{d}{d \ln \nu} \delta H_{2}^{(1)}\left((p+q)^{2}, q^{2}, u, \mu, \nu\right)=\frac{\alpha_{s}(\mu)}{4 \pi} \gamma_{m}^{(0)} H_{2}^{(0)}\left((p+q)^{2}, q^{2}, u\right), \quad \gamma_{m}^{(0)}=6 C_{F},
$$

and the consistency condition valid to all orders of $\alpha_{s}$

$$
\delta H_{2}^{(1)}\left((p+q)^{2}, q^{2}, u, \mu, \nu=\mu\right)=0 .
$$

It is then straightforward to find

$$
\delta H_{2}^{(1)}\left((p+q)^{2}, q^{2}, u, \mu, \nu\right)=\left[\frac{\alpha_{s}(\mu)}{4 \pi} \gamma_{m}^{(0)} \ln \left(\frac{\nu}{\mu}\right)\right] H_{2}^{(0)}\left((p+q)^{2}, q^{2}, u\right) .
$$


According to the RG evolution equation of the twist-two photon LCDA [54]

$$
\frac{d}{d \ln \mu}\left[\chi(\mu)\langle\bar{q} q\rangle(\mu) \phi_{\gamma}(u, \mu)\right]=\int_{0}^{1} d u^{\prime} V\left(u, u^{\prime}\right)\left[\chi(\mu)\langle\bar{q} q\rangle(\mu) \phi_{\gamma}\left(u^{\prime}, \mu\right)\right],
$$

and expanding the renormalization kernel in terms of the strong coupling constant

$$
\begin{aligned}
V\left(u, u^{\prime}\right) & =\sum_{n}\left(\frac{\alpha_{s}(\mu)}{4 \pi}\right)^{n+1} V^{(n)}\left(u, u^{\prime}\right), \\
V^{(0)}\left(u, u^{\prime}\right) & =2 C_{F}\left[\frac{1-u}{1-u^{\prime}} \frac{\theta\left(u-u^{\prime}\right)}{u-u^{\prime}}+\frac{u}{u^{\prime}} \frac{\theta\left(u^{\prime}-u\right)}{u^{\prime}-u}\right]_{+}-C_{F} \delta\left(u-u^{\prime}\right),
\end{aligned}
$$

we can readily verify that the perturbative QCD factorization formula (3.33) for the twisttwo hadronic photon contribution to the correlation function (2.5) is indeed independent of the factorization scale $\mu$ at one loop as expected. More explicitly, we obtain

$$
\frac{d \Pi_{\mu, \mathrm{NLO}}^{\mathrm{tw} 2}}{d \ln \mu}=\mathcal{O}\left(\alpha_{s}^{2}\right) .
$$

Apparently, the parametrically large logarithms of $m_{Q} / \Lambda_{\mathrm{QCD}}$ entering the hardcollinear factorization formula (3.33) cannot be eliminated by making use of the common value of the factorization scale $\mu$. The NLL resummation of such logarithms to all orders can be achieved by solving the evolution equation for the twist-two photon LCDA at two loops. Expanding $\phi_{\gamma}(u, \mu)$ in a series of Gegenbauer polynomials [22]

$$
\phi_{\gamma}(u, \mu)=6 u(1-u) \sum_{n}^{\infty} b_{n}(\mu) C_{n}^{3 / 2}(2 u-1),
$$

the general solution to the above-mentioned RG equation can be constructed as follows [55]

$$
\begin{aligned}
\chi(\mu)\langle\bar{q} q\rangle(\mu) b_{n}(\mu)= & E_{T, n}^{\mathrm{NLO}}\left(\mu, \mu_{0}\right)\left[\chi\left(\mu_{0}\right)\langle\bar{q} q\rangle\left(\mu_{0}\right) b_{n}\left(\mu_{0}\right)\right] \\
& +\frac{\alpha_{s}(\mu)}{4 \pi} \sum_{k=0}^{n-2} E_{T, n}^{\mathrm{LO}}\left(\mu, \mu_{0}\right) d_{T, n}^{k}\left(\mu, \mu_{0}\right)\left[\chi\left(\mu_{0}\right)\langle\bar{q} q\rangle\left(\mu_{0}\right) b_{k}\left(\mu_{0}\right)\right],
\end{aligned}
$$

where the manifest expressions of the evolution functions $E_{T, n}^{(\mathrm{N}) \mathrm{LO}}$ and $d_{T, n}^{k}$ can be found in [11], and $n, k$ are non-negative even integers. The obtained hard-collinear factorization formula for the correlation function (2.5) with the RG improvement can be further written as

$$
\begin{aligned}
\Pi_{\mu, \mathrm{NLL}}^{\mathrm{tw} 2}(p, q)= & -g_{\mathrm{em}} e_{q} \chi(\mu)\langle\bar{q} q\rangle(\mu) \epsilon_{\mu p q \eta^{*}} \\
& \sum_{n} b_{n}(\mu)\left[G_{n}^{(0)}\left((p+q)^{2}, q^{2}\right)+\frac{\alpha_{s}(\mu) C_{F}}{4 \pi} G_{n}^{(1)}\left((p+q)^{2}, q^{2}, \mu\right)\right],
\end{aligned}
$$

where the perturbative kernels $G_{n}^{(i)}(i=0,1)$ are defined by the convolution integrals

$$
\begin{aligned}
G_{n}^{(0)}\left((p+q)^{2}, q^{2}\right) & =\int_{0}^{1} d u \frac{6 u(1-u) C_{n}^{3 / 2}(2 u-1)}{\bar{u}(p+q)^{2}+u q^{2}-m_{Q}^{2}+i 0} \\
G_{n}^{(1)}\left((p+q)^{2}, q^{2}\right) & =\int_{0}^{1} d u \frac{6 u(1-u) C_{n}^{3 / 2}(2 u-1)}{\bar{u}(p+q)^{2}+u q^{2}-m_{Q}^{2}+i 0} \frac{H_{2}^{(1)}\left((p+q)^{2}, q^{2}, u, \mu\right)}{H_{2}^{(0)}\left((p+q)^{2}, q^{2}, u\right)} .
\end{aligned}
$$


In analogy to the construction of the tree-level LCSR (3.14) for the twist-two resolved photon contribution, we proceed to derive the spectral representation of the resummation improved factorization formula (3.43)

$$
\begin{aligned}
\Pi_{\mu, \mathrm{NLL}}^{\mathrm{tw} 2}(p, q)= & -g_{\mathrm{em}} e_{q} \chi(\mu)\langle\bar{q} q\rangle(\mu) \epsilon_{\mu p q} \eta^{*} \int_{0}^{\infty} d s_{1} \int_{0}^{\infty} d s_{2} \frac{1}{\left[s_{1}-(p+q)^{2}-i 0\right]\left[s_{2}-q^{2}-i 0\right]} \\
& \times\left[\rho_{0}^{\mathrm{tw} 2}\left(s_{1}, s_{2}\right)+\frac{\alpha_{s}(\mu) C_{F}}{4 \pi} \rho_{1}^{\mathrm{tw} 2}\left(s_{1}, s_{2}\right)\right] .
\end{aligned}
$$

Following the discussion presented in [43], it turns out to be sufficient to extract the NLO spectral density $\rho_{1}^{\text {tw2 }}\left(s_{1}, s_{2}\right)$ with the asymptotic photon distribution amplitude $\phi_{\gamma}^{\text {asy }}(u, \mu)=6 u(1-u)$ by discarding the subdominant effects due to the non-vanishing higher Gegenbauer moments. Taking advantage of the spectral representations of various complex functions displayed in appendix B, it is then straightforward to derive the analytical expression of the double spectral density

$$
\begin{aligned}
m_{Q}^{2} \rho_{1}^{\mathrm{tw} 2}\left(s_{1}, s_{2}\right)= & \left\{\left[\left[\varrho_{\mathrm{I}}(r, \sigma)+\varrho_{\mathrm{II}}(r, \sigma) \ln r+\varrho_{\mathrm{III}}(r, \sigma)\left(\ln ^{2} r-\pi^{2}\right)\right] \delta^{(2)}(r-1)\right.\right. \\
& \left.+\left[\varrho_{\mathrm{II}}(r, \sigma)+2 \varrho_{\mathrm{III}}(r, \sigma) \ln r\right] \frac{d^{3}}{d r^{3}} \ln |1-r|\right\} \theta\left(s_{1}-m_{Q}^{2}\right) \theta\left(s_{2}-m_{Q}^{2}\right),
\end{aligned}
$$

where we have introduced the invariant functions

$$
\begin{aligned}
\varrho_{\mathrm{I}}(r, \sigma)= & \frac{(r+1)}{\sigma^{3}}\left\{6 r \sigma^{2}\left[2 \ln \left(\frac{\mu^{2}}{m_{Q}^{2}}\right)+\frac{3}{2} \ln \left(\frac{\nu^{2}}{\mu^{2}}\right)+3 \operatorname{Li}_{2}\left(-\frac{\sigma}{r+1}\right)+\operatorname{Li}_{2}\left(-\frac{r \sigma}{r+1}\right)\right]\right. \\
& +6 r \sigma^{2} \ln \left(\frac{\sigma}{r+1}\right)\left[\ln \left(\frac{\sigma+r+1}{r+1}\right)+\ln \left(\frac{r \sigma+r+1}{r+1}\right)\right] \\
& +3(r+1)[2 r(\sigma+1)+5 \sigma+2] \ln \left(\frac{\sigma+r+1}{r+1}\right) \\
& -\frac{3 r \sigma^{2}}{(r+\sigma+1)(r \sigma+r+1)}\left[9 r \sigma^{2}+(7 r+10)(r+1) \sigma+8(r+1)^{2}\right] \ln \left(\frac{\sigma}{r+1}\right) \\
& \left.+\frac{\sigma}{r+\sigma+1}\left[\left(\left(3+2 \pi^{2}\right) r+6\right) \sigma^{2}+\left(\left(9+2 \pi^{2}\right) r-6\right)(r+1) \sigma-6(r+1)^{2}\right]\right\} \\
& +6 m_{Q}^{4}\left[4+3 \ln \left(\frac{\mu^{2}}{m_{Q}^{2}}\right)\right] \frac{d}{d m_{Q}^{2}}\left[\frac{1}{m_{Q}^{2}} \frac{r(r+1)}{\sigma}\right], \\
\varrho_{\mathrm{II}}(r, \sigma)= & 3 r(r+1)\left[\frac{r}{r \sigma+r+1}+\frac{2}{r+\sigma+1}-\frac{2}{\sigma} \ln \left(\frac{\sigma+r+1}{r \sigma+r+1}\right)\right], \\
\varrho_{\mathrm{III}}(r, \sigma)= & -\frac{6 r(r+1)}{\sigma},
\end{aligned}
$$

with two new dimensionless variables defined by

$$
r=\frac{s_{1}-m_{Q}^{2}}{s_{2}-m_{Q}^{2}}, \quad \sigma=\frac{s_{1}}{m_{Q}^{2}}+\frac{s_{2}}{m_{Q}^{2}}-2 .
$$


The obtained NLL LCSR for the twist-two hadronic photon correction to the radiative heavy meson decay form factor can be written as

$$
\begin{aligned}
& f_{P} f_{V} \mu_{P} m_{V} g_{M^{*} M \gamma}^{(\mathrm{tw} 2, \mathrm{NLL})} \exp \left[-\left(\frac{m_{V}^{2}+m_{P}^{2}}{M^{2}}\right)\right] \\
& =e_{q} \chi(\mu)\langle\bar{q} q\rangle(\mu) \iint_{\Sigma} d s_{1} d s_{2} \exp \left[-\left(\frac{s_{1}+s_{2}}{M^{2}}\right)\right]\left[\rho_{0}^{\mathrm{tw} 2}\left(s_{1}, s_{2}\right)+\frac{\alpha_{s}(\mu) C_{F}}{4 \pi} \rho_{1}^{\mathrm{tw} 2}\left(s_{1}, s_{2}\right)\right] .
\end{aligned}
$$

Since the NLL spectral density $\rho_{1}^{\mathrm{tw} 2}\left(s_{1}, s_{2}\right)$ is not concentrated at $s_{1}=s_{2}$, the result of the dispersion integral entering (3.51) now depends on the precise shape of the duality region $\Sigma$ in the $\left(s_{1}, s_{2}\right)$ plane, in contrast to the tree-level LCSR displayed in (3.14). Employing the duality boundary $s_{1}+s_{2} \leq 2 s_{0}$ implemented in [43] and performing the Jacobi transformation introduced in (3.50), we can readily derive

$$
\begin{aligned}
& f_{P} f_{V} \mu_{P}(\nu) m_{V} g_{M^{*} M \gamma}^{(\mathrm{tw} 2, \mathrm{NLL})} \exp \left[-\left(\frac{m_{V}^{2}+m_{P}^{2}}{M^{2}}\right)\right] \\
& =e_{q} \chi(\mu)\langle\bar{q} q\rangle(\mu)\left\{-\frac{M^{2}}{2}\left[\exp \left(-\frac{2 m_{Q}^{2}}{M^{2}}\right)-\exp \left(-\frac{2 s_{0}}{M^{2}}\right)\right] \phi_{\gamma}\left(\frac{1}{2}, \mu\right)\right. \\
& \quad+\frac{\alpha_{s}(\mu) C_{F}}{4 \pi}\left[\int_{2 m_{Q}^{2}}^{2 s_{0}} d s \exp \left(-\frac{s}{M^{2}}\right) \mathcal{F}^{(\mathrm{tw} 2)}\left(\frac{s}{m_{Q}^{2}}-2\right)\right. \\
& \left.\left.\quad+3 m_{Q}^{2}\left(4+3 \ln \frac{\mu^{2}}{m_{Q}^{2}}\right) \exp \left(-\frac{2 m_{Q}^{2}}{M^{2}}\right)\right]\right\}
\end{aligned}
$$

where the explicit expression of $\mathcal{F}^{(\mathrm{tw} 2)}(\sigma)$ is given by

$$
\begin{aligned}
\mathcal{F}^{(\mathrm{tw} 2)}(\sigma)= & -3\left\{\ln \left(\frac{\mu^{2}}{m_{Q}^{2}}\right)+\frac{3}{4} \ln \left(\frac{\nu^{2}}{\mu^{2}}\right)-\mathrm{Li}_{2}(-\sigma)-\mathrm{Li}_{2}(-\sigma-1)+2 \mathrm{Li}_{2}\left(-\frac{\sigma}{2}\right)+\frac{\pi^{2}}{12}\right. \\
& +\ln \left(\frac{\sigma}{2}\right) \ln \left(\frac{\sigma+2}{2}\right)-\ln (\sigma+1) \ln (\sigma+2)+\frac{2(\sigma+1)^{2}}{(\sigma+2)^{3}} \ln (\sigma+1) \\
& \left.-\frac{7 \sigma^{3}+50 \sigma^{2}+100 \sigma+64}{4(\sigma+2)^{3}} \ln \left(\frac{\sigma}{2}\right)-\frac{1}{2} \ln \left(\frac{\sigma+2}{2}\right)-\frac{11 \sigma^{2}+28 \sigma+24}{8(\sigma+2)^{2}}\right\} .
\end{aligned}
$$

This together with the one-loop expression of the double spectral function $\rho_{1}^{\text {tw2 }}\left(s_{1}, s_{2}\right)$ constitutes one of the major technical results of this paper. We further verify that the obtained LCSR (3.52) for the twist-two resolved photon correction to the magnetic coupling $g_{M^{*} M \gamma}$ is independent of the factorization scale $\mu$ and the renormalization scale $\nu$ of the local QCD current $J_{5}=\bar{Q} \gamma_{5} q$ with the asymptotic photon LCDA at one loop.

\section{The higher twist LCSR for the resolved photon effects}

The objective of this section is to compute the higher twist corrections to the magnetic coupling from the two-particle and three-particle photon distribution amplitudes at tree level, up to the twist-four accuracy. In analogy to the derivation of the twist-two LCSR 
for the hadronic photon correction detailed in section 3, we will need to demonstrate the hard-collinear factorization formulae of the vacuum-to-photon correlation function (2.5) for the subleading twist contributions by employing the OPE technique and the background field formalism with the default power counting scheme (2.8).

\subsection{Two-particle higher twist corrections}

The two-particle higher twist corrections to the correlation function (2.5) can be obtained by keeping the subleading power terms in the light-cone expansion of the heavy-quark propagator

$$
\Pi_{\mu}(p, q) \supset \int d^{4} x \int \frac{d^{4} k}{(2 \pi)^{4}} \frac{e^{-i(p+q+k) \cdot x}}{k^{2}-m_{Q}^{2}}\left\langle\gamma\left(p, \eta^{*}\right)\left|\bar{q}(x)\left(\sigma_{\mu \nu} k^{\nu}+i m_{Q} \gamma_{\mu \perp}\right) \gamma_{5} q(0)\right| 0\right\rangle .
$$

According to the definitions of the twist-three and twist-four photon LCDA presented in appendix $\mathrm{C}$, the corresponding hard-collinear factorization formula can be written as

$$
\Pi_{\mu}(p, q) \supset \frac{e_{q} g_{\mathrm{em}}}{4} \epsilon_{\mu p q \eta^{*}} \int_{0}^{1} d u\left\{\frac{K_{2}^{2 \mathrm{PHT}}\left((p+q)^{2}, q^{2}, u\right)}{\left[\bar{u}(p+q)^{2}+u q^{2}-m_{Q}^{2}\right]^{2}}+\frac{K_{3}^{2 \mathrm{PHT}}\left((p+q)^{2}, q^{2}, u\right)}{\left[\bar{u}(p+q)^{2}+u q^{2}-m_{Q}^{2}\right]^{3}}\right\},
$$

where the two coefficient functions $K_{2(3)}^{2 \mathrm{PHT}}$ are given by

$$
\begin{aligned}
& K_{2}^{2 \mathrm{PHT}}\left((p+q)^{2}, q^{2}, u\right)=2 m_{Q} f_{3 \gamma}(\mu) \psi^{(a)}(u, \mu)-\langle\bar{q} q\rangle(\mu) \mathbb{A}(u, \mu), \\
& K_{3}^{2 \mathrm{PHT}}\left((p+q)^{2}, q^{2}, u\right)=2 m_{Q}^{2}\langle\bar{q} q\rangle(\mu) \mathbb{A}(u, \mu) .
\end{aligned}
$$

The dispersion representation of the tree-level factorization formula (4.2) can be further derived with the general expression for the double spectral densities of the invariant amplitudes

$$
\begin{aligned}
& \frac{1}{\pi^{2}} \operatorname{Im}_{s_{1}} \operatorname{Im}_{s_{2}} \int_{0}^{1} d u \frac{\varphi(u, \mu)}{\left[\bar{u} s_{1}+u s_{2}-m_{Q}^{2}+i 0\right]^{n}} \\
& =\frac{1}{\Gamma(n)} \frac{d^{n-1}}{\left(d m_{Q}^{2}\right)^{n-1}} \sum_{k} \frac{(-1)^{k+1} c_{k}(\mu)}{\Gamma(k+1)}\left(s_{1}-m_{Q}^{2}\right)^{k} \delta^{(k)}\left(s_{1}-s_{2}\right),
\end{aligned}
$$

where we have employed the Taylor expansion of $\varphi(u, \mu)$ at $u=0$

$$
\varphi(u, \mu)=\sum_{k} c_{k}(\mu) u^{k} .
$$

Confronting the obtained spectral representation of (4.2) with the double hadronic dispersion relation (2.22), implementing the continuum subtraction with the parton-hadron duality approximation and performing the double Borel transformation $(p+q)^{2} \rightarrow M^{2}$, $q^{2} \rightarrow M^{2}$, we derive the LCSR for the two-particle higher twist corrections to the magnetic coupling $g_{M^{*} M \gamma}$

$$
\begin{aligned}
& f_{P} f_{V} \mu_{P} m_{V} g_{M^{*} M \gamma}^{(2 \mathrm{PHT})} \exp \left[-\left(\frac{m_{V}^{2}+m_{P}^{2}}{M^{2}}\right)\right] \\
& =\frac{e_{q}}{2}\left[m_{Q} f_{3 \gamma}(\mu) \psi^{(a)}\left(\frac{1}{2}, \mu\right)+\left(\frac{m_{Q}^{2}}{M^{2}}+\frac{1}{2}\right)\langle\bar{q} q\rangle(\mu) \mathbb{A}\left(\frac{1}{2}, \mu\right)\right] \exp \left(-\frac{2 m_{Q}^{2}}{M^{2}}\right) .
\end{aligned}
$$


As can be understood from (4.4), the obtained subleading twist sum rules (4.6) at tree level are also independent of the specific shape of the duality boundary due to the vanishing spectral function at $s_{1} \neq s_{2}$. Several remarks on the newly derived LCSR (4.6) are in order.

- Based upon the canonical behaviour of the two-particle photon LCDA $\psi^{(a)}$ and $\mathbb{A}$ as well as the power counting scheme for the sum rule parameters displayed in (2.28), we can derive the scaling law of each individual term appearing in (4.6)

$$
\left.g_{M^{*} M \gamma}^{(2 \mathrm{PHT})}\right|_{\psi^{(a)}} \sim \mathcal{O}\left(\frac{1}{\Lambda_{\mathrm{QCD}}}\right),\left.\quad g_{M^{*} M \gamma}^{(2 \mathrm{PHT})}\right|_{\mathbb{A}} \sim \mathcal{O}\left(\frac{1}{\omega_{0}}\right) .
$$

In comparison with the scaling behaviour of the twist-two hadronic photon contribution displayed in (3.15), the two-particle twist-three and twist-four corrections to the magnetic coupling $M^{*} M \gamma$ are suppressed by one and two power(s) of $\Lambda_{\mathrm{QCD}} / \omega_{0}$, respectively.

- Applying the VMD model, the nonperturbative parameter $f_{3 \gamma}$ characterizing the twist-three contribution can be expressed in terms of the relevant $\rho$-meson couplings [22]

$$
f_{3 \gamma}^{\mathrm{VMD}} \simeq-f_{\rho}^{2} \zeta_{3, \rho}, \quad \zeta_{3, \rho}(1 \mathrm{GeV})=0.10 \pm 0.05
$$

The appearance of the strong suppression factor $\zeta_{3, \rho}$ in (4.8) implies that the twoparticle higher twist contributions to the coupling $g_{M^{*} M \gamma}$ will be numerically dominated by the twist-four term entering (4.6).

\subsection{Three-particle higher twist corrections}

We turn to compute the subleading power corrections to the heavy meson magnetic coupling from the three-particle higher twist photon distribution amplitudes by employing the LCSR technique. To achieve this goal, we derive the corresponding higher twist factorization formula of the vacuum-to-photon correlation function (2.5) with the aid of the light-cone expansion for the quark propagator in the background gluon/photon field $[44,56]$

$$
\begin{aligned}
\langle 0|\mathrm{~T}\{\bar{q}(x), q(0)\}| 0\rangle \supset & i g_{s} \int_{0}^{\infty} \frac{d^{4} k}{(2 \pi)^{4}} e^{-i k \cdot x} \int_{0}^{1} d u\left[\frac{u x_{\mu} \gamma_{\nu}}{k^{2}-m_{q}^{2}}-\frac{\left(\not k+m_{q}\right) \sigma_{\mu \nu}}{2\left(k^{2}-m_{q}^{2}\right)^{2}}\right] G^{\mu \nu}(u x) \quad \text { (4.9) } \\
& +i e_{q} g_{\mathrm{em}} \int_{0}^{\infty} \frac{d^{4} k}{(2 \pi)^{4}} e^{-i k \cdot x} \int_{0}^{1} d u\left[\frac{u x_{\mu} \gamma_{\nu}}{k^{2}-m_{q}^{2}}-\frac{\left(\not k+m_{q}\right) \sigma_{\mu \nu}}{2\left(k^{2}-m_{q}^{2}\right)^{2}}\right] F^{\mu \nu}(u x) .
\end{aligned}
$$

Computing the tree-level diagrams displayed in figure 4 and making use of the definitions for the three-particle photon LCDA collected in appendix C, we find

$$
\Pi_{\mu}(p, q) \supset g_{\mathrm{em}}\langle\bar{q} q\rangle(\mu) \epsilon_{\mu p q \eta^{*}} \int_{0}^{1} d u \int\left[\mathcal{D} \alpha_{i}\right] \frac{e_{q} K_{2, G}^{3 \mathrm{PHT}}\left(\alpha_{i}, u\right)+e_{Q} K_{2, \gamma}^{3 \mathrm{PHT}}\left(\alpha_{i}, u\right)}{\left[\bar{\alpha}_{u}(p+q)^{2}+\alpha_{u} q^{2}-m_{Q}^{2}+i 0\right]^{2}},
$$

where we have introduced the following short-handed notations

$$
\alpha_{u}=\alpha_{q}+u \alpha_{g}, \quad \bar{\alpha}_{u}=1-\alpha_{u},
$$




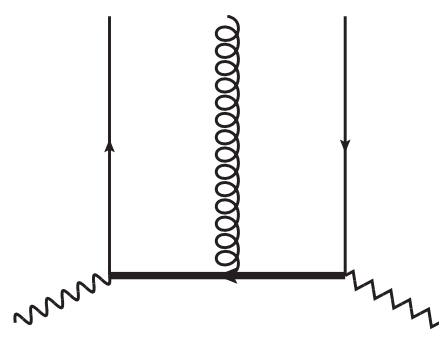

(a)

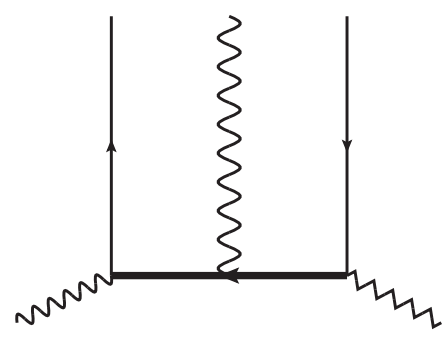

(b)

Figure 4. Diagrammatic representation of the three-particle higher twist corrections to the vacuum-to-photon correlation function (2.5) at tree level.

and the integration measure is given by

$$
\int\left[\mathcal{D} \alpha_{i}\right]=\int_{0}^{1} d \alpha_{q} \int_{0}^{1} d \alpha_{\bar{q}} \int_{0}^{1} d \alpha_{g} \delta\left(1-\alpha_{q}-\alpha_{\bar{q}}-\alpha_{g}\right)
$$

The two invariant functions $K_{2, G(\gamma)}^{3 \mathrm{PHT}}$ can be expressed in terms of the three-particle twistfour distribution amplitudes

$$
\begin{aligned}
& K_{2, G}^{3 \mathrm{PHT}}=S\left(\alpha_{i}, \mu\right)+T_{1}\left(\alpha_{i}, \mu\right)-T_{2}\left(\alpha_{i}, \mu\right)+(2 u-1)\left[\tilde{S}\left(\alpha_{i}, \mu\right)-T_{3}\left(\alpha_{i}, \mu\right)+T_{4}\left(\alpha_{i}, \mu\right)\right], \\
& K_{2, \gamma}^{3 \mathrm{PHT}}=S_{\gamma}\left(\alpha_{i}, \mu\right)+(2 u-1) T_{4}^{\gamma}\left(\alpha_{i}, \mu\right) .
\end{aligned}
$$

The spectral representation of the hard-collinear factorization formula (4.10) can be constructed with the master formula for computing the double spectral density

$$
\begin{aligned}
& \frac{1}{\pi^{2}} \operatorname{Im}_{s_{1}} \operatorname{Im}_{s_{2}} \int_{0}^{1} d u \int\left[\mathcal{D} \alpha_{i}\right] \frac{u^{m} \Phi\left(\alpha_{i}, \mu\right)}{\left[\bar{\alpha}_{u} s_{1}+\alpha_{u} s_{2}-m_{Q}^{2}+i 0\right]^{n}} \\
& =\frac{1}{\Gamma(n)} \frac{d^{n-1}}{\left(d m_{Q}^{2}\right)^{n-1}} \sum_{k} \frac{(-1)^{k+1} h_{m, k}(\mu)}{\Gamma(k+1)}\left(s_{1}-m_{Q}^{2}\right)^{k} \delta^{(k)}\left(s_{1}-s_{2}\right),
\end{aligned}
$$

where the renormalization-scale dependent coefficient $h_{m, k}(\mu)$ arises from the series expansion of the "effective" photon distribution amplitude $\widehat{\Phi}\left(\alpha_{u}, m, \mu\right)$ defined by

$$
\widehat{\Phi}\left(\alpha_{u}, m, \mu\right)=\int_{0}^{\alpha_{u}} d \alpha_{q} \int_{\alpha_{u}-\alpha_{q}}^{1-\alpha_{q}} d \alpha_{g} \frac{\left(\alpha_{u}-\alpha_{q}\right)^{m}}{\alpha_{g}^{m+1}} \Phi\left(\alpha_{q}, 1-\alpha_{q}-\alpha_{g}, \alpha_{g}, \mu\right) .
$$

More explicitly,

$$
\widehat{\Phi}\left(\alpha_{u}, m, \mu\right)=\sum_{k} h_{m, k}(\mu) \alpha_{u}^{k}
$$

We can then readily derive the tree-level LCSR for the three-particle higher twist correc- 
tions to the magnetic coupling $M^{*} M \gamma$ of our interest

$$
\begin{aligned}
& f_{P} f_{V} \mu_{P} m_{V} g_{M^{*} M \gamma}^{(3 \mathrm{PHT})} \exp \left[-\left(\frac{m_{V}^{2}+m_{P}^{2}}{M^{2}}\right)\right] \\
& =-\langle\bar{q} q\rangle(\mu)\left\{e _ { q } \left[\widehat{S}\left(\frac{1}{2}, 0, \mu\right)+\widehat{T}_{1}\left(\frac{1}{2}, 0, \mu\right)-\widehat{T}_{2}\left(\frac{1}{2}, 0, \mu\right)-\widehat{\tilde{S}}\left(\frac{1}{2}, 0, \mu\right)+\widehat{T}_{3}\left(\frac{1}{2}, 0, \mu\right)\right.\right. \\
& \left.-\widehat{T}_{4}\left(\frac{1}{2}, 0, \mu\right)+2 \widehat{\widetilde{S}}\left(\frac{1}{2}, 1, \mu\right)-2 \widehat{T}_{3}\left(\frac{1}{2}, 1, \mu\right)+2 \widehat{T}_{4}\left(\frac{1}{2}, 1, \mu\right)\right] \\
& \left.\quad+e_{Q}\left[\widehat{S}_{\gamma}\left(\frac{1}{2}, 0, \mu\right)-\widehat{T}_{4}^{\gamma}\left(\frac{1}{2}, 0, \mu\right)+2 \widehat{T}_{4}^{\gamma}\left(\frac{1}{2}, 1, \mu\right)\right]\right\} \exp \left(-\frac{2 m_{Q}^{2}}{M^{2}}\right) .
\end{aligned}
$$

Employing the power counting scheme (2.28) we can establish the scaling laws for the separate three-particle twist-four contributions from the quark-anti-quark-gluon and quarkanti-quark-photon distribution amplitudes

$$
\left.\left.g_{M^{*} M \gamma}^{(3 \mathrm{PHT})}\right|_{\bar{q} q G} \sim g_{M^{*} M \gamma}^{(3 \mathrm{PHT})}\right|_{\bar{q} q \gamma} \sim \mathcal{O}\left(\frac{1}{m_{Q}}\right)
$$

which suffer from the double suppression factor of $\Lambda_{\mathrm{QCD}}^{2} /\left(m_{Q} \omega_{0}\right)$ compared with the twisttwo resolved photon contribution shown in (3.15). Consequently, the three-particle higher twist corrections to the radiative bottom-meson decay form factors are expected to be of minor importance numerically.

Putting all the different pieces together, the final expression for the heavy meson magnetic coupling computed from the LCSR method with the collinear photon distribution amplitudes can be written as

$$
g_{M^{*} M \gamma}=g_{M^{*} M \gamma}^{(\mathrm{per})}+g_{M^{*} M \gamma}^{(\mathrm{tw} 2, \mathrm{NLL})}+g_{M^{*} M \gamma}^{(2 \mathrm{PHT})}+g_{M^{*} M \gamma}^{(3 \mathrm{PHT})},
$$

where the analytical expressions of the individual terms appearing in the right-hand side of (4.19) are already displayed in (2.26), (3.52), (4.6) and (4.17), respectively. We make several comments on the higher-order corrections to the $M^{*} M \gamma$ couplings accomplished in this work.

- It is apparent that constructing the desired LCSR for the heavy meson magnetic coupling from the vacuum-to-photon correlation function (2.5) is achieved with the parton-hadron duality approximation for both the vector and pseudoscalar heavymeson channels. The resulting LCSR predictions for the $M^{*} M \gamma$ couplings are therefore less accurate than the ones for the semileptonic heavy-to-light transition form factors (see, for instance $[57,58]$ and references therein).

- In order to verify whether the higher-order radiative corrections can alleviate the double suppression mechanism for the three-particle twist-four contributions at tree level, it would be interesting to derive the NLO LCSR for the subleading twist hadronic photon contributions to the magnetic couplings for future development. 


\section{$5 \quad$ Numerical analysis}

Having at our disposal the improved LCSR for the heavy meson magnetic coupling, we are now ready to explore the phenomenological implications of the newly obtained power corrections from the two-particle and three-particle photon distribution amplitudes at twistfour. We further perform an exploratory comparison of our results for the $M^{*} M \gamma$ couplings with other estimates based upon different QCD techniques and with the available experimental measurements from the CLEO, BESIII, and BaBar Collaborations.

\subsection{Theory inputs}

To perform the numerical study of the derived LCSR (4.19) for the radiative heavy meson decay form factors, we start discussing the numerical input values of both the QCD and hadronic parameters as collected in table 1. We employ the renormalized light-quark masses in the $\overline{\mathrm{MS}}$ scheme at $\mu=2 \mathrm{GeV}$ determined from the lattice QCD calculations with $N_{f}=4\left(N_{f}\right.$ is the number of active quark flavors at the renormalization scale $\left.\mu\right)$ [59], which are also in good agreement with the $\mathrm{N}^{4} \mathrm{LO}$ QCDSR predictions [64] based upon the five-loop computation of the two-point correlation function of the scalar strangenesschanging quark currents (see [65] for an independent determination of the strange-quark mass from the pseudoscalar sum rules). In addition, the $\overline{\mathrm{MS}}$ charm-quark mass determined from the $\mathrm{N}^{3} \mathrm{LO}$ relativistic QCDSR with the vector-current correlation function [60] and the $\overline{\mathrm{MS}}$ bottom-quark mass from the non-relativistic sum rules with high moments of $6 \leq n \leq 15$ at $\mathrm{N}^{3} \mathrm{LO}$ [61] will be implemented in the numerical analysis. We further adopt the lattice QCD calculations of the decay constants for the pseudoscalar heavy mesons with $N_{f}=2+1+1$ dynamical flavours summarized in the Flavour Lattice Averaging Group (FLAG) review [62], which are compatible with the three-loop QCDSR predictions [66] with higher theoretical uncertainties. The QCD decay constants of the vector heavy-light mesons are taken from the lattice determinations with the gauge configurations produced by European Twisted Mass Collaboration (ETMC) at $N_{f}=2+1+1$ [63]. It is interesting to notice that the predicted heavy-quark spin/flavour symmetry breaking effects for the decay

constant ratios $f_{D_{(s)}^{*}} / f_{D_{(s)}}>1$ in the charm sector differ from the ones $f_{B_{(s)}^{*}} / f_{B_{(s)}}<1$ in the bottom sector.

The fundamental nonperturbative functions entering the sum rules (4.19) for the heavy meson magnetic couplings are the two-particle and three-particle collinear photon distribution amplitudes at twist-four. The explicit expressions of these hadronic quantities at NLO in the conformal expansion can be found in [22] (see also [8] for a summary). Among the eleven nonperturbative parameters in total, $\langle\bar{q} q\rangle, \chi, f_{3 \gamma}, \kappa, \kappa^{+}$are defined by the QCD matrix elements of local operators without the covariant derivative acting on the quark/gluon fields. We use the averaged $N_{f}=2+1+1$ determinations of the low-energy constant $\langle\bar{q} q\rangle$ at the renormalization scale $2 \mathrm{GeV}$ from the lattice QCD calculations [62], which are consistent with the interval determined from the chiral perturbation theory (ChPT) relations $[57,67]$. An updated prediction of the magnetic susceptibility of the quark condensate from the QCDSR method including the radiative correction to the corresponding partonic spectral density presented in [22] will be adopted in what follows. We further 


\begin{tabular}{|c|c|c|c|c|c|}
\hline Parameter & Value & Ref. & Parameter & Value & Ref. \\
\hline $\bar{m}_{u}(2 \mathrm{GeV})$ & $2.32 \pm 0.10 \mathrm{MeV}$ & {$[59]$} & $\bar{m}_{c}\left(\bar{m}_{c}\right)$ & $1.288 \pm 0.020 \mathrm{GeV}$ & {$[60]$} \\
\hline $\bar{m}_{d}(2 \mathrm{GeV})$ & $4.71 \pm 0.09 \mathrm{MeV}$ & {$[59]$} & $\bar{m}_{b}\left(\bar{m}_{b}\right)$ & $4.193_{-0.035}^{+0.022} \mathrm{GeV}$ & {$[61]$} \\
\hline $\bar{m}_{s}(2 \mathrm{GeV})$ & $92.9 \pm 0.7 \mathrm{MeV}$ & {$[59]$} & & & \\
\hline$m_{D^{ \pm}}$ & $1869.65 \pm 0.05 \mathrm{MeV}$ & {$[59]$} & $m_{B^{ \pm}}$ & $5279.33 \pm 0.13 \mathrm{MeV}$ & {$[59]$} \\
\hline$m_{D^{0}}$ & $1864.83 \pm 0.05 \mathrm{MeV}$ & {$[59]$} & $m_{B^{0}}$ & $5279.64 \pm 0.13 \mathrm{MeV}$ & {$[59]$} \\
\hline$m_{D_{s}}$ & $1968.34 \pm 0.07 \mathrm{MeV}$ & {$[59]$} & $m_{B_{s}}$ & $5366.88 \pm 0.17 \mathrm{MeV}$ & {$[59]$} \\
\hline$m_{D^{* \pm}}$ & $2010.26 \pm 0.05 \mathrm{MeV}$ & {$[59]$} & $m_{B^{* \pm}}$ & $5324.70 \pm 0.22 \mathrm{MeV}$ & {$[59]$} \\
\hline$m_{D^{* 0}}$ & $2006.85 \pm 0.05 \mathrm{MeV}$ & {$[59]$} & $m_{B^{* 0}}$ & $5324.86 \pm 0.22 \mathrm{MeV}$ & {$[59]$} \\
\hline$m_{D_{s}^{*}}$ & $2112.2 \pm 0.4 \mathrm{MeV}$ & {$[59]$} & $m_{B_{s}^{*}}$ & $5415.4_{-1.5}^{+1.8} \mathrm{MeV}$ & {$[59]$} \\
\hline$\left.f_{D}\right|_{N_{f}=2+1+1}$ & $212.0 \pm 0.7 \mathrm{MeV}$ & {$[62]$} & $\left.f_{B}\right|_{N_{f}=2+1+1}$ & $190.0 \pm 1.3 \mathrm{MeV}$ & {$[62]$} \\
\hline$\left.f_{D_{s}}\right|_{N_{f}=2+1+1}$ & $249.9 \pm 0.5 \mathrm{MeV}$ & {$[62]$} & $\left.f_{B_{s}}\right|_{N_{f}=2+1+1}$ & $230.3 \pm 1.3 \mathrm{MeV}$ & {$[62]$} \\
\hline$\left.f_{D^{*}}\right|_{N_{f}=2+1+1}$ & $223.5 \pm 8.4 \mathrm{MeV}$ & {$[63]$} & $\left.f_{B^{*}}\right|_{N_{f}=2+1+1}$ & $185.9 \pm 7.2 \mathrm{MeV}$ & {$[63]$} \\
\hline$\left.f_{D_{s}^{*}}\right|_{N_{f}=2+1+1}$ & $268.8 \pm 6.6 \mathrm{MeV}$ & {$[63]$} & $\left.f_{B_{s}^{*}}\right|_{N_{f}=2+1+1}$ & $223.1 \pm 5.4 \mathrm{MeV}$ & {$[63]$} \\
\hline$\langle\bar{q} q\rangle(1 \mathrm{GeV})$ & $-(266 \pm 21 \mathrm{MeV})^{3}$ & {$[62]$} & $\kappa(1 \mathrm{GeV})$ & $0.2 \pm 0.2$ & {$[2,22]$} \\
\hline$\chi(1 \mathrm{GeV})$ & $(3.15 \pm 0.3) \mathrm{GeV}^{-2}$ & {$[22]$} & $\kappa^{+}(1 \mathrm{GeV})$ & 0 & {$[2,22]$} \\
\hline$b_{2}(1 \mathrm{GeV})$ & $0.07 \pm 0.07$ & {$[22]$} & $\zeta_{1}(1 \mathrm{GeV})$ & $0.4 \pm 0.4$ & {$[2,22]$} \\
\hline$f_{3 \gamma}(1 \mathrm{GeV})$ & $-(4 \pm 2) \times 10^{-3} \mathrm{GeV}^{2}$ & {$[2,22]$} & $\zeta_{1}^{+}(1 \mathrm{GeV})$ & 0 & {$[2,22]$} \\
\hline$\omega_{\gamma}^{V}(1 \mathrm{GeV})$ & $3.8 \pm 1.8$ & {$[2,22]$} & $\zeta_{2}^{+}(1 \mathrm{GeV})$ & 0 & {$[2,22]$} \\
\hline$\omega_{\gamma}^{A}(1 \mathrm{GeV})$ & $-2.1 \pm 1.0$ & {$[22]$} & & & \\
\hline$\left\{s_{0}, M^{2}\right\}$ & $\{6.0 \pm 0.5,4.5 \pm 1.0\} \mathrm{GeV}^{2}$ & $D^{*} D \gamma$ & $\left\{s_{0}, M^{2}\right\}$ & $\{34.0 \pm 1.0,18.0 \pm 3.0\} \mathrm{GeV}^{2}$ & $B^{*} B \gamma$ \\
\hline$\left\{s_{0}, M^{2}\right\}$ & $\{7.0 \pm 0.5,4.5 \pm 1.0\} \mathrm{GeV}^{2}$ & $D_{s}^{*} D_{s} \gamma$ & $\left\{s_{0}, M^{2}\right\}$ & $\{36.0 \pm 1.0,18.0 \pm 3.0\} \mathrm{GeV}^{2}$ & $B_{s}^{*} B_{s} \gamma$ \\
\hline
\end{tabular}

Table 1. Numerical input values of the theory parameters employed in the improved LCSR predictions for the $M^{*} M \gamma$ couplings with the subleading twist corrections.

take the values of the normalization constant $f_{3 \gamma}$, parameterizing the three-body local vacuum-to-photon matrix element $\left\langle\gamma\left(p, \eta^{*}\right)\left|\bar{q} g_{s} \tilde{G}_{\alpha \beta} \gamma_{\rho} \gamma_{5} q\right| 0\right\rangle$, estimated from the VMD approximation, which are also confirmed by the QCDSR calculation independently [22]. In addition, $\kappa, \kappa^{+}$and the six remaining nonperturbative parameters (i.e., $b_{2}, \omega_{\gamma}^{V}, \omega_{\gamma}^{A}, \zeta_{1}$, $\left.\zeta_{1}^{+}, \zeta_{2}^{+}\right)$entering the "P"-wave terms in the conformal expansion of the photon LCDA are taken from the numerical evaluations obtained in [2]. As discussed in detail in [22], the parameter $\zeta_{2}$ entering the three-particle twist-four photon distribution amplitudes can be expressed in terms of $\zeta_{1}$ and $\zeta_{2}^{+}$, thanks to the Ferrara-Grillo-Parisi-Gatto theorem [68]. The renormalization scale dependence of the twist-three and twist-four parameters at the leading-logarithmic (LL) approximation can be derived by solving the corresponding oneloop evolution equations [22, 69].

The determinations of the Borel parameter $M^{2}$ and the effective threshold $s_{0}$ can be achieved by applying the standard criteria imposed in the LCSR calculations: the smallness of subleading twist contributions in the OPE, and simultaneously, the suppression of higher state contributions (see [34] for a modern review). The obtained intervals of 


\begin{tabular}{|c||c|c|c||c|c|c|}
\hline & $D^{*+} D^{+} \gamma$ & $D^{* 0} D^{0} \gamma$ & $D_{s}^{*+} D_{s}^{+} \gamma$ & $B^{*+} B^{+} \gamma$ & $B^{* 0} B^{0} \gamma$ & $B_{s}^{* 0} B_{s}^{0} \gamma$ \\
\hline$g_{M^{*} M \gamma}^{(\mathrm{er})}\left(\mathrm{GeV}^{-1}\right)$ & -0.032 & 0.73 & 0.024 & 0.84 & -0.56 & -0.47 \\
\hline$g_{M^{*} M \gamma}^{(\mathrm{tw}, \mathrm{LL})}\left(\mathrm{GeV}^{-1}\right)$ & -0.50 & 0.99 & -0.41 & 0.96 & -0.48 & -0.37 \\
\hline$g_{M^{*} M \gamma}^{(\mathrm{tw} 2, \mathrm{NLL})}\left(\mathrm{GeV}^{-1}\right)$ & -0.45 & 0.89 & -0.36 & 0.79 & -0.40 & -0.31 \\
\hline$g_{M^{*} M \gamma}^{(2 \mathrm{PHT})}\left(\mathrm{GeV}^{-1}\right)$ & 0.18 & -0.37 & 0.14 & -0.18 & 0.089 & 0.067 \\
\hline$g_{M^{*} M \gamma}^{(3 \mathrm{PHT})}\left(\mathrm{GeV}^{-1}\right)$ & 0.14 & 0.23 & 0.11 & -0.017 & -0.036 & -0.027 \\
\hline$g_{M^{*} M \gamma}\left(\mathrm{GeV}^{-1}\right)$ & -0.15 & 1.48 & -0.079 & 1.44 & -0.91 & -0.74 \\
\hline
\end{tabular}

Table 2. The numerical results of all separate terms entering the obtained LCSR (4.19) for the $M^{*} M \gamma$ couplings with the central values of theory inputs.

theses "internal" sum rule parameters presented in table 1 are in agreement with the previous choices adopted in the LCSR analysis of heavy-to-light decay form factors [57, 70]. Furthermore, we will vary the factorization scale $\mu$ appearing in the NLL LCSR of the twist-two hadronic photon contribution (3.52) in the range $[1.0,2.0] \mathrm{GeV}$ around the default value $\bar{m}_{c}\left(\bar{m}_{c}\right)$ for the magnetic couplings $D_{q}^{*} D_{q} \gamma$ and in $\left[m_{b} / 2,2 m_{b}\right]$ around $\bar{m}_{b}\left(\bar{m}_{b}\right)$ for the counterpart bottom-meson couplings. The renormalization scale for the QCD pseudoscalar current will be further taken as $\nu=m_{Q}[46,52]$.

\subsection{Theory predictions for radiative heavy meson form factors}

We are now in a position to investigate the numerical impacts of the perturbative QCD corrections and the subleading twist corrections to the resolved photon contributions for the heavy meson magnetic couplings. It is apparent from table 2 that the twist-two hadronic photon corrections to the bottom-meson couplings $B_{q}^{*} B_{q} \gamma$ are numerically comparable to the corresponding "point-like" photon contributions and the QCD radiative corrections will reduce the tree-level twist-two predictions by approximately an amount of $20 \%$. In addition, such long-distance twist-two corrections turn out to be the most significant contributions for the charm-meson magnetic couplings, particularly for the radiative $D^{*+} \rightarrow D^{+} \gamma$ and $D_{s}^{*+} \rightarrow D_{s}^{+} \gamma$ decay form factors, where the leading power "point-like" photon contributions are numerically suppressed due to the strong cancellation between the photon radiation off the charm and light quarks as already mentioned in section 2. The $\mathrm{SU}(3)$-flavour symmetry breaking effect between the two magnetic couplings $B^{* 0} B^{0} \gamma$ and $B_{s}^{* 0} B_{s}^{0} \gamma$ is evaluated to be approximately $\mathcal{O}(20 \%)$ based upon the LCSR technique, in analogy to the ones for the semileptonic $B$-meson form factors [45, 46]. Furthermore, our predictions for the two-particle and three-particle higher twist corrections to the $B_{q}^{*} B_{q} \gamma$ couplings at tree level imply that the subleading twist terms appearing in the bottom-meson sum rules are indeed of minor phenomenological importance, in contrary to the observed patterns for the counterpart charm-meson couplings as displayed in table 2.

We proceed to present the individual uncertainties for the predicted heavy meson magnetic couplings due to variations of the input parameters in table 3 , where the total theory 


\begin{tabular}{|c||c|c|c||c|c|c|}
\hline & $\begin{array}{c}g_{D^{*+} D^{+} \gamma} \\
\left(\mathrm{GeV}^{-1}\right)\end{array}$ & $\begin{array}{c}g_{D^{* 0} D^{0} \gamma} \\
\left(\mathrm{GeV}^{-1}\right)\end{array}$ & $\begin{array}{c}g_{D_{s}^{*+} D_{s}^{+} \gamma}\left(\mathrm{GeV}^{-1}\right) \\
\text { central value }\end{array}$ & $\begin{array}{c}g_{B^{*+} B^{+} \gamma} \\
\left(\mathrm{GeV}^{-1}\right)\end{array}$ & $\begin{array}{c}g_{B^{* 0} B^{0} \gamma} \\
\left(\mathrm{GeV}^{-1}\right)\end{array}$ & $\begin{array}{c}g_{B_{s}^{* 0} B_{s}^{0} \gamma} \\
\left(\mathrm{GeV}^{-1}\right)\end{array}$ \\
\hline$\Delta f_{P}$ & \pm 0.15 & 1.48 & -0.079 & 1.44 & -0.91 & -0.74 \\
\hline$\Delta f_{V}$ & \pm 0.01 & \pm 0.05 & \pm 0.002 & \pm 0.05 & \pm 0.03 & \pm 0.02 \\
\hline$\Delta m_{Q}$ & \pm 0.00 & \pm 0.02 & ${ }_{-0.004}^{+0.005}$ & ${ }_{-0.04}^{+0.06}$ & ${ }_{-0.04}^{+0.02}$ & ${ }_{-0.03}^{+0.02}$ \\
\hline$\Delta s_{0}$ & \pm 0.00 & ${ }_{-0.09}^{+0.07}$ & \pm 0.004 & ${ }_{-0.06}^{+0.05}$ & \pm 0.04 & ${ }_{-0.02}^{+0.03}$ \\
\hline$\Delta M^{2}$ & ${ }_{-0.03}^{+0.07}$ & \pm 0.01 & ${ }_{-0.019}^{+0.050}$ & \pm 0.02 & ${ }_{-0.02}^{+0.01}$ & \pm 0.02 \\
\hline$\Delta \mu$ & ${ }_{-0.02}^{+0.00}$ & ${ }_{-0.00}^{+0.05}$ & ${ }_{-0.019}^{+0.001}$ & ${ }_{-0.04}^{+0.00}$ & ${ }_{-0.00}^{+0.02}$ & ${ }_{-0.00}^{+0.02}$ \\
\hline$\Delta\langle\bar{q} q\rangle$ & ${ }_{-0.05}^{+0.04}$ & ${ }_{-0.19}^{+0.22}$ & ${ }_{-0.039}^{+0.033}$ & ${ }_{-0.14}^{+0.17}$ & ${ }_{-0.09}^{+0.08}$ & ${ }_{-0.07}^{+0.06}$ \\
\hline$\Delta \chi$ & \pm 0.04 & \pm 0.08 & \pm 0.034 & \pm 0.08 & \pm 0.04 & \pm 0.03 \\
\hline$\Delta b_{2}$ & \pm 0.05 & \pm 0.10 & \pm 0.042 & \pm 0.07 & \pm 0.04 & \pm 0.03 \\
\hline$\Delta f_{3 \gamma}$ & \pm 0.03 & \pm 0.06 & \pm 0.024 & \pm 0.03 & \pm 0.01 & \pm 0.03 \\
\hline$\Delta \omega_{\gamma}^{V}$ & \pm 0.02 & \pm 0.03 & \pm 0.014 & \pm 0.01 & \pm 0.01 & \pm 0.01 \\
\hline$\Delta \kappa$ & \pm 0.01 & \pm 0.03 & \pm 0.011 & \pm 0.01 & \pm 0.01 & \pm 0.00 \\
\hline$\Delta_{\text {tot }}$ & ${ }_{-0.10}^{+0.11}$ & ${ }_{-0.27}^{+0.29}$ & ${ }_{-0.078}^{+0.086}$ & ${ }_{-0.20}^{+0.22}$ & ${ }_{-0.13}^{+0.12}$ & ${ }_{-0.10}^{+0.09}$ \\
\hline
\end{tabular}

Table 3. Summary of the individual theory uncertainties for the heavy meson magnetic couplings $M^{*} M \gamma$ predicted from the LCSR (4.19). The negligibly small uncertainties due to variations of the remaining input parameters are not displayed here explicitly, but are already taken into account in the determinations of the total errors $\Delta_{\text {tot }}$.

uncertainties obtained by adding all separate uncertainties in quadrature are further shown for completeness. Evidently, the dominant theory uncertainties of the LCSR predictions for the bottom-meson couplings arise from the variations of the quark condensate density $\langle\bar{q} q\rangle$ as well as its magnetic susceptibility $\chi$. As expected, the LCSR predictions for the charm-meson magnetic couplings suffer from larger theory uncertainties than the ones for the corresponding bottom-meson couplings. It needs to be pointed out further that the substantial uncertainties for the two couplings $D^{*+} D^{+} \gamma$ and $D_{s}^{*+} D_{s}^{+} \gamma$ can be attributed to the almost complete cancellation of the two different pieces entering the "point-like" photon contribution (2.26), which are classified in terms of the electric-charge factors $e_{q}$ and $e_{Q}$ respectively.

Finally, we compare our predictions of the heavy meson magnetic couplings with the previous theory determinations obtained from different QCD techniques and phenomenological models in table 4 , where the $\mathrm{HH} \chi \mathrm{PT}$ results including both the $\mathcal{O}\left(m_{q}^{1 / 2}\right)$ corrections to the photon radiation off the light quarks and the subleading power corrections to the 


\begin{tabular}{|c||c|c|c||c|c|c|}
\hline & $\begin{array}{c}g_{D^{*+} D^{+} \gamma} \\
\left(\mathrm{GeV}^{-1}\right)\end{array}$ & $\begin{array}{c}g_{D^{* 0} D^{0} \gamma} \\
\left(\mathrm{GeV}^{-1}\right)\end{array}$ & $\begin{array}{c}g_{D_{s}^{*+} D_{s}^{+} \gamma} \\
\left(\mathrm{GeV}^{-1}\right)\end{array}$ & $\begin{array}{c}g_{B^{*+} B^{+} \gamma} \\
\left(\mathrm{GeV}^{-1}\right)\end{array}$ & $\begin{array}{c}g_{B^{* 0} B^{0} \gamma} \\
\left(\mathrm{GeV}^{-1}\right)\end{array}$ & $\begin{array}{c}g_{B_{s}^{* 0} B_{s}^{0} \gamma} \\
\left(\mathrm{GeV}^{-1}\right)\end{array}$ \\
\hline this work & $-0.15_{-0.10}^{+0.11}$ & $1.48_{-0.27}^{+0.29}$ & $-0.079_{-0.078}^{+0.086}$ & $1.44_{-0.20}^{+0.22}$ & $-0.91_{-0.13}^{+0.12}$ & $-0.74_{-0.10}^{+0.09}$ \\
\hline HH $\chi$ PT [24] & $-0.27 \pm 0.05$ & $2.19 \pm 0.11$ & $0.041 \pm 0.056$ & $1.45 \pm 0.11$ & $-1.01 \pm 0.05$ & $-0.70 \pm 0.06$ \\
\hline HQET+VMD [35] & $-0.29_{-0.11}^{+0.19}$ & $1.60_{-0.45}^{+0.35}$ & $-0.19_{-0.08}^{+0.19}$ & $0.99_{-0.23}^{+0.19}$ & $-0.58_{-0.10}^{+0.12}$ & - \\
\hline HQET+CQM [71] & $-0.38_{-0.06}^{+0.05}$ & $1.91 \pm 0.09$ & - & $1.45_{-0.12}^{+0.11}$ & $-0.82_{-0.05}^{+0.06}$ & - \\
\hline Lattice QCD [32] & $-0.2 \pm 0.3$ & $2.0 \pm 0.6$ & - & - & - & - \\
\hline LCSR [21] & $-0.50 \pm 0.12$ & $1.52 \pm 0.25$ & - & $1.68 \pm 0.17$ & $-0.85 \pm 0.17$ & - \\
\hline QCDSR [20] & $-0.19_{-0.02}^{+0.03}$ & $0.62 \pm 0.03$ & $-0.20 \pm 0.03$ & - & - & - \\
\hline RQM [72] & $-0.44 \pm 0.06$ & $2.15 \pm 0.11$ & $-0.19 \pm 0.03$ & $1.66 \pm 0.11$ & $-0.93 \pm 0.05$ & $0.65 \pm 0.03$ \\
\hline experiment [16-18] & $-0.47 \pm 0.06$ & $1.77 \pm 0.03$ & - & - & - & - \\
\hline
\end{tabular}

Table 4. Comparisons of the LCSR calculations for the heavy-meson magnetic couplings with the previous determinations and the available experimental data.

photon coupling to the heavy quarks are derived from the following formula [24]

$$
g_{M^{*} M \gamma}=\frac{e_{Q}}{m_{Q}}\left(1+\frac{2}{3} \frac{\bar{\Lambda}}{m_{Q}}\right)+e_{q} \beta+\delta \mu_{q}^{(\ell)} .
$$

The non-perturbative HQET parameter $\bar{\Lambda}$ characterizing the canonical size of the power corrections to the heavy-quark mass limit is estimated to be $\bar{\Lambda} \simeq 0.50 \mathrm{GeV}$ from the two-point sum rule method $[73,74]$. The effective coupling $\beta$ appearing in the $\mathrm{HH} \chi \mathrm{PT}$ Lagrangian $[26,27]$ has been determined to be $\beta=(3.41 \pm 0.16) \mathrm{GeV}^{-1}[75]$, consistent with the predictions from the nonrelativistic quark model [24]. The explicit expressions of the SU(3)-flavour symmetry breaking terms $\delta \mu_{q}^{(\ell)}$ generated by the pion and kaon loops are given by [24]

$$
\delta \mu_{u}^{(\ell)}=-\frac{g_{\pi}^{2} m_{K}}{4 \pi f_{K}^{2}}-\frac{g_{\pi}^{2} m_{\pi}}{4 \pi f_{\pi}^{2}}, \quad \delta \mu_{d}^{(\ell)}=-\frac{g_{\pi}^{2} m_{\pi}}{4 \pi f_{\pi}^{2}}, \quad \delta \mu_{s}^{(\ell)}=-\frac{g_{\pi}^{2} m_{K}}{4 \pi f_{K}^{2}} .
$$

We will employ the interval of the strong coupling $g_{\pi}=0.57 \pm 0.01$ extracted from the experimental measurements for the decay width of $D^{*+} \rightarrow D^{0} \pi^{+}[76,77]$. Apparently, our LCSR determinations of the heavy meson magnetic couplings are in reasonable agreement with the achieved $\mathrm{HH} \chi \mathrm{PT}$ predictions within the theory uncertainties shown in table 4 .

Instead of computing the hadronic matrix elements of the electromagnetic currents of the light-flavour quarks from the $\mathrm{HH} \chi \mathrm{PT}$ technique, the VMD approximation and the covariant quark model $(\mathrm{CQM})$ are employed to evaluate the second and third terms $e_{q} \beta+\delta \mu_{q}^{(\ell)}$ entering the expression for the heavy meson magnetic coupling (5.1) in [35] and [71], respectively. In general we observe a fair agreement of the resulting predictions from three different approaches, with the exceptions of $g_{D^{*+} D^{+\gamma}}$ and $g_{B^{* 0} B^{0} \gamma}$. Moreover, the available lattice QCD results of the charm-meson magnetic couplings $g_{D^{*+} D^{+} \gamma}$ and $g_{D^{* 0} D^{0} \gamma}$ [32] obtained by employing the gauge field configurations from the QCDSF Collaboration [78] are 
compatible with our LCSR calculations. Confronting the previous LCSR predictions including the two-particle higher twist corrections [21] with the three-point QCDSR estimates at tree level [20] already reveals some tensions of the determined intervals for $g_{D^{*+} D^{+\gamma}}$ and $g_{D^{* 0} D^{0} \gamma}$, which could be traced back to the unaccounted subleading twist corrections from the collinear photon distribution amplitudes in the above-mentioned LCSR and the systematic uncertainty of the classical sum rule method due to the contamination from the non-diagonal transitions of the ground state to excited states [79]. In addition, the predicted bottom-meson magnetic couplings from the relativistic quark model (RQM) [72] are in excellent agreement with our LCSR results. However, the aforementioned RQM predictions for the counterpart charm-meson magnetic couplings are consistently higher in magnitude than our determinations. We also mention in passing that our prediction for the $B^{* 0} B \gamma$ coupling is consistent with the one determined in [80].

We further collect the extracted values of $g_{D^{*+} D^{+\gamma}}$ from the CLEO data on the branching ratio of the radiative decay $D^{*+} \rightarrow D^{+} \gamma$ [16] and the Particle Data Group (PDG) average of $\Gamma\left(D^{*+}\right)=83.4 \pm 1.8 \mathrm{keV}$ [59] in table 4, where the displayed interval for the neutral charm-meson magnetic coupling is obtained from the experimental measurements of $\mathcal{B R}\left(D^{* 0} \rightarrow D^{0} \gamma\right)$ [59] and the estimated result of the total decay width $\Gamma\left(D^{* 0}\right)=55.4 \pm 1.4 \mathrm{keV}$ by applying the well-known isospin symmetry relations of the following strong coupling constants [44]

$$
g_{D^{*+} D^{0} \pi^{+}}=-\sqrt{2} g_{D^{*+} D^{+} \pi^{0}}=\sqrt{2} g_{D^{* 0} D^{0} \pi^{0}} .
$$

As far as the magnitude is concerned, our computations of the two couplings $g_{D^{*+} D^{+\gamma}}$ and $g_{D^{* 0} D^{0} \gamma}$ yield somewhat lower values than determined from the experimental measurements of the corresponding radiative decay widths. It would be interesting to investigate whether such discrepancies can be resolved by taking into account the NLO QCD corrections to the "point-like" photon contributions in the LCSR framework.

\section{Conclusion}

In the present paper we have computed the twist-two hadronic photon corrections to the radiative heavy meson decay form factors at the NLL accuracy with the aid of the LCSR technique. The resummation improved hard-collinear factorization formula for the vacuumto-photon correlation function defined with the two interpolating currents for the vector and pseudoscalar heavy mesons was established by applying the evanescent operator approach and the two-loop RG equation of the twist-two photon distribution amplitude. We derived the double spectral representation of the resulting QCD factorization formula and subsequently construed the desired LCSR for the twist-two resolved photon contributions to the magnetic couplings $M^{*} M \gamma$ analytically by implementing the parton-hadron duality ansatz and the double Borel transformation. The subleading twist corrections from both the two-particle and three-particle photon LCDA up to and including twist-four were further evaluated at tree level from the same LCSR method, taking advantage of the background field formalism. The newly determined double spectral densities for the subleading 
twist contributions enabled us to perform the continuum subtractions analytically in constructing the higher-twist sum rules on the light-cone.

Exploring the obtained LCSR for the magnetic $M^{*} M \gamma$ couplings numerically we observed that the twist-two hadronic photon corrections to the bottom-meson couplings are comparable to the counterpart "point-like" photon contributions despite of the $\Lambda_{\mathrm{QCD}} / \omega_{0}$ suppression. In particular, such structure-dependent hadronic corrections gave rise to the dominant contributions to the two magnetic couplings $D^{*+} D^{+} \gamma$ and $D_{s}^{*+} D_{s}^{+} \gamma$, confirming the previous observations concluded from the three-point sum rule calculation [20] and the $\mathrm{HH} \chi \mathrm{PT}$ analysis [24]. Moreover, the predicted NLL QCD corrections to the twist-two resolved photon contributions of the bottom-meson magnetic couplings can generate approximately $20 \%$ reduction to the corresponding tree-level determinations. We further noticed that the subleading twist contributions to the charm-meson couplings from the two-particle and three-particle photon distribution amplitudes turned out to be more pronounced than the counterpart effects for the bottom-meson magnetic couplings. Confronting our LCSR predictions with various evaluations from the diverse QCD techniques generally led to a fair agreement for the obtained values of the $M^{*} M \gamma$ couplings within the theory uncertainties.

Developing the LCSR for the heavy-meson radiative decay form factors beyond the current work can be pursued further in different directions. First, computing the NLO QCD corrections to the leading power "point-like" photon contributions will be in high demand in order to achieve a better understanding of the observed $g_{D^{* 0} D^{0} \gamma}$ tension between the LCSR predictions and the CLEO measurements. The technical challenges of constructing such NLO sum rules arise from both the two-loop computations of the vacuum-to-photon correlation function (2.5) and the analytical determinations of the double spectral densities entering the dispersion representation of the derived QCD factorization formula. Second, it will be of both technical and conceptual interest to compute the perturbative QCD corrections to the higher-twist contributions at twist-four systematically in the LCSR framework. Extracting the hard matching coefficients appearing in the higher-twist factorization formula for the correlation function (2.5) at NLO will be complicated by the nontrivial infrared subtractions due to the renormalization mixing of the different light-ray collinear operators. Third, updating the non-perturbative parameters in the conformal expansion of the photon distribution amplitudes with the standard QCDSR approach will be also of phenomenological importance to pin down the theory uncertainties for the LCSR calculations of the magnetic $M^{*} M \gamma$ couplings.

\section{Acknowledgments}

C.D.L is supported in part by the National Natural Science Foundation of China (NSFC) with Grant No. 11521505 and 11621131001. Y.M.W acknowledges support from the National Youth Thousand Talents Program, the Youth Hundred Academic Leaders Program of Nankai University, the National Natural Science Foundation of China with Grant No. 11675082 and 11735010, and the Natural Science Foundation of Tianjin with Grant No. 19JCJQJC61100. The work of Y.B.W is supported in part by the NSFC with Grant No. 11847238. Y.M.W also would like to thank Martin Beneke for the warm hospitality during his visit at Technical University Munich, Germany. 


\section{A Useful one-loop integrals}

We collect in this appendix the analytical results of various one-loop Feynman integrals for evaluating the NLO QCD corrections to the twist-two resolved photon contributions displayed in section 3 .

$$
\begin{aligned}
& I_{a}=\int[\mathcal{D} \ell] \frac{m_{Q}^{2}}{\left[(\ell+u p)^{2}+i 0\right]\left[(\ell-\bar{u} p-q)^{2}-m_{Q}^{2}+i 0\right]\left[\ell^{2}+i 0\right]} \\
& =\frac{1}{r_{2}-r_{1}}\left\{\left[\frac{1}{\epsilon}+\ln \frac{\mu^{2}}{m_{Q}^{2}}-\ln \left[\left(1-r_{1}\right)\left(1-r_{2}\right)\right]\right] \ln \frac{1-r_{1}}{1-r_{2}}+\operatorname{Li}_{2}\left(r_{2}\right)-\operatorname{Li}_{2}\left(r_{1}\right)\right\} \text {, } \\
& I_{a, \alpha}=\int[\mathcal{D} \ell] \frac{m_{Q}^{2} \ell_{\alpha}}{\left[(\ell+u p)^{2}+i 0\right]\left[(\ell-\bar{u} p-q)^{2}-m_{Q}^{2}+i 0\right]\left[\ell^{2}+i 0\right]} \\
& =I_{A}(u p)_{\alpha}+I_{B}(p+q)_{\alpha}, \\
& I_{a, \alpha \beta}=\int[\mathcal{D} \ell] \frac{\ell_{\alpha}^{\perp} \ell_{\beta}^{\perp}}{\left[(\ell+u p)^{2}+i 0\right]\left[(\ell-\bar{u} p-q)^{2}-m_{Q}^{2}+i 0\right]\left[\ell^{2}+i 0\right]} \\
& =\frac{g_{\alpha \beta}^{\perp}}{4}\left\{\frac{1}{\epsilon}+\ln \frac{\mu^{2}}{m_{Q}^{2}}+\frac{1}{r_{2}-r_{1}}\left[\frac{\left(1-r_{1}\right)^{2}}{r_{1}} \ln \left(1-r_{1}\right)-\frac{\left(1-r_{2}\right)^{2}}{r_{2}} \ln \left(1-r_{2}\right)\right]+3\right\}, \\
& I_{b, \alpha}=\int[\mathcal{D} \ell] \frac{m_{Q}^{2} \ell_{\alpha}}{\left[(\ell-\bar{u} p)^{2}+i 0\right]\left[(\ell-\bar{u} p-q)^{2}-m_{Q}^{2}+i 0\right]\left[\ell^{2}+i 0\right]} \\
& =-\left.I_{A}\right|_{r_{2} \rightarrow r_{3}}(\bar{u} p)_{\alpha}+\left.I_{B}\right|_{r_{2} \rightarrow r_{3}} q_{\alpha}, \\
& I_{c}=\int[\mathcal{D} \ell] \frac{1}{\left[(\ell-\bar{u} p-q)^{2}-m_{Q}^{2}+i 0\right]\left[\ell^{2}+i 0\right]} \\
& =\frac{1}{\epsilon}+\ln \frac{\mu^{2}}{m_{Q}^{2}}-\left(1-\frac{1}{r_{1}}\right) \ln \left(1-r_{1}\right)+2 \text {, } \\
& I_{c, \alpha}=\int[\mathcal{D} \ell] \frac{\ell_{\alpha}}{\left[(\ell-\bar{u} p-q)^{2}-m_{Q}^{2}+i 0\right]\left[\ell^{2}+i 0\right]} \\
& =\frac{1}{2}\left[\frac{1}{\epsilon}+\ln \frac{\mu^{2}}{m_{Q}^{2}}-\left(\frac{1-r_{1}}{r_{1}}\right)^{2} \ln \left(1-r_{1}\right)+2-\frac{1}{r_{1}}\right](\bar{u} p+q)_{\alpha}, \\
& I_{d, \alpha}=\int[\mathcal{D} \ell] \frac{m_{Q}^{2} \bar{n} \cdot \ell \ell_{\alpha}}{\left[(\ell+u p)^{2}+i 0\right]\left[(\ell-\bar{u} p)^{2}+i 0\right]\left[(\ell-\bar{u} p-q)^{2}-m_{Q}^{2}+i 0\right]\left[\ell^{2}+i 0\right]} \\
& =\frac{1}{n \cdot p}\left[I_{b, \alpha}-I_{a, \alpha}\right] \text {, }
\end{aligned}
$$

with

$$
\begin{aligned}
I_{A} & =\frac{1}{r_{2}-r_{1}}\left[\left(r_{1}-1\right) I_{a}-2 r_{2} I_{B}+I_{c}\right], \\
I_{B} & =\frac{1}{r_{2}-r_{1}}\left[\frac{1-r_{1}}{r_{1}} \ln \left(1-r_{1}\right)-\frac{1-r_{2}}{r_{2}} \ln \left(1-r_{2}\right)\right] .
\end{aligned}
$$


The integration measure is defined as

$$
\int[\mathcal{D} \ell]=\frac{(4 \pi)^{2}}{i}\left[\frac{\mu^{2} e^{\gamma_{E}}}{4 \pi}\right]^{\epsilon} \int \frac{d^{D} \ell}{(2 \pi)^{D}},
$$

and the dimensionless parameters $r_{1}=(\bar{u} p+q)^{2} / m_{Q}^{2}, r_{2}=(p+q)^{2} / m_{Q}^{2}$ and $r_{3}=q^{2} / m_{Q}^{2}$ are further introduced for brevity.

\section{B Master formulae for the spectral representations}

Here we collect the necessary identities for computing the double spectral density appearing in the dispersion representation of the NLL QCD factorization formula of the correlation function (2.5) at the twist-two accuracy.

$$
\begin{aligned}
& \frac{1}{\pi} \operatorname{Im}_{r_{3}} \int_{r_{2}}^{r_{3}} d \rho \frac{\ln (1-\rho)}{1-\rho} f(\rho)=-\int_{r_{2}}^{r_{3}} d \rho\left[\frac{\theta(\rho-1)}{1-\rho}\right]_{+} f(\rho)+\ln \left(r_{3}-1\right) f(1), \\
& \frac{1}{\pi} \operatorname{Im}_{r_{3}} \int_{r_{2}}^{r_{3}} d \rho \frac{\operatorname{Li}_{2}(\rho)}{1-\rho} f(\rho)=\int_{r_{2}}^{r_{3}} d \rho\left[\frac{\theta(\rho-1) \ln \rho}{1-\rho}\right]_{+} f(\rho) \\
& +\left[\operatorname{Li}_{2}\left(1-r_{3}\right)+\frac{\pi^{2}}{6}\right] f(1) \\
& \frac{1}{\pi} \operatorname{Im}_{r_{3}} \int_{r_{2}}^{r_{3}} d \rho \frac{\ln ^{2}(1-\rho)}{1-\rho} f(\rho)=-2 \int_{r_{2}}^{r_{3}} d \rho\left[\frac{\theta(\rho-1) \ln (\rho-1)}{1-\rho}\right]_{+} f(\rho) \\
& +\left[\ln ^{2}\left(r_{3}-1\right)-\frac{\pi^{2}}{3}\right] f(1) \\
& \frac{1}{\pi} \operatorname{Im}_{r_{3}} \int_{r_{2}}^{r_{3}} d \rho \frac{\ln \left(1-r_{3}\right)}{1-\rho} f(\rho)=-\int_{r_{2}}^{r_{3}} d \rho\left[\frac{\theta(1-\rho)}{1-\rho}+\frac{\theta(\rho-1)}{1-\rho}\right]_{+} f(\rho) \\
& +\ln \frac{\left(r_{3}-1\right)^{2}}{1-r_{2}} f(1) \\
& \frac{1}{\pi} \operatorname{Im}_{r_{3}} \int_{r_{2}}^{r_{3}} d \rho \frac{\operatorname{Li}_{2}\left(r_{3}\right)}{1-\rho} f(\rho)=\int_{r_{2}}^{r_{3}} d \rho\left[\frac{\theta(1-\rho)}{1-\rho}+\frac{\theta(\rho-1)}{1-\rho}\right]_{+} \ln r_{3} f(\rho) \\
& -\left\{\operatorname{Li}_{2}\left(1-r_{3}\right)+\ln r_{3} \ln \frac{\left(r_{3}-1\right)^{2}}{1-r_{2}}-\frac{\pi^{2}}{6}\right\} f(1), \\
& \frac{1}{\pi} \operatorname{Im}_{r_{3}} \int_{r_{2}}^{r_{3}} d \rho \frac{\ln ^{2}\left(1-r_{3}\right)}{1-\rho} f(\rho)=-2 \int_{r_{2}}^{r_{3}} d \rho\left[\frac{\theta(1-\rho)}{1-\rho}+\frac{\theta(\rho-1)}{1-\rho}\right]_{+} \ln \left(r_{3}-1\right) f(\rho) \\
& +\left\{\ln \left(r_{3}-1\right) \ln \frac{\left(r_{3}-1\right)^{3}}{\left(1-r_{2}\right)^{2}}-\pi^{2}\right\} f(1)
\end{aligned}
$$

where the "plus" function is defined by

$$
\int_{r_{2}}^{r_{3}} d \rho[g(\rho)]_{+} f(\rho)=\int_{r_{2}}^{r_{3}} d \rho g(\rho)[f(\rho)-f(1)]
$$

and the Heaviside step function $\theta\left(r_{3}-1\right)$ has been suppressed on the right-hand sides of (B.1)-(B.6). 


\section{Photon distribution amplitudes}

We summarize the operator-level definitions of the two-particle and three-particle photon distribution amplitudes up to and including the twist-four accuracy, following the systematic classification detailed in [22], and taking this opportunity correct several misprints in the previous expressions displayed in appendix B of [8].

$$
\begin{aligned}
& \left\langle\gamma\left(p, \eta^{*}\right)\left|\bar{q}(x) W_{c}(x, 0) \sigma_{\alpha \beta} q(0)\right| 0\right\rangle \\
& =-i g_{\mathrm{em}} Q_{q}\langle\bar{q} q\rangle(\mu)\left(p_{\beta} \eta_{\alpha}^{*}-p_{\alpha} \eta_{\beta}^{*}\right) \int_{0}^{1} d z e^{i z p \cdot x}\left[\chi(\mu) \phi_{\gamma}(z, \mu)+\frac{x^{2}}{16} \mathbb{A}(z, \mu)\right] \\
& -\frac{i}{2} g_{\mathrm{em}} Q_{q} \frac{\langle\bar{q} q\rangle(\mu)}{q \cdot x}\left(x_{\beta} \eta_{\alpha}^{*}-x_{\alpha} \eta_{\beta}^{*}\right) \int_{0}^{1} d z e^{i z p \cdot x} h_{\gamma}(z, \mu) . \\
& \left\langle\gamma\left(p, \eta^{*}\right)\left|\bar{q}(x) W_{c}(x, 0) \gamma_{\alpha} q(0)\right| 0\right\rangle=g_{\mathrm{em}} Q_{q} f_{3 \gamma}(\mu) \eta_{\alpha}^{*} \int_{0}^{1} d z e^{i z p \cdot x} \psi^{(v)}(z, \mu) . \\
& \left\langle\gamma\left(p, \eta^{*}\right)\left|\bar{q}(x) W_{c}(x, 0) \gamma_{\alpha} \gamma_{5} q(0)\right| 0\right\rangle \\
& =\frac{g_{\mathrm{em}}}{4} Q_{q} f_{3 \gamma}(\mu) \epsilon_{\alpha \beta \rho \tau} p^{\rho} x^{\tau} \eta^{* \beta} \int_{0}^{1} d z e^{i z p \cdot x} \psi^{(a)}(z, \mu) . \\
& \left\langle\gamma\left(p, \eta^{*}\right)\left|\bar{q}(x) W_{c}(x, 0) g_{s} G_{\alpha \beta}(v x) q(0)\right| 0\right\rangle \\
& =-i g_{\mathrm{em}} Q_{q}\langle\bar{q} q\rangle(\mu)\left(p_{\beta} \eta_{\alpha}^{*}-p_{\alpha} \eta_{\beta}^{*}\right) \int\left[\mathcal{D} \alpha_{i}\right] e^{i\left(\alpha_{q}+v \alpha_{g}\right) p \cdot x} S\left(\alpha_{i}, \mu\right) \text {. } \\
& \left\langle\gamma\left(p, \eta^{*}\right)\left|\bar{q}(x) W_{c}(x, 0) g_{s} \widetilde{G}_{\alpha \beta}(v x) i \gamma_{5} q(0)\right| 0\right\rangle \\
& =i g_{\mathrm{em}} Q_{q}\langle\bar{q} q\rangle(\mu)\left(p_{\beta} \eta_{\alpha}^{*}-p_{\alpha} \eta_{\beta}^{*}\right) \int\left[\mathcal{D} \alpha_{i}\right] e^{i\left(\alpha_{q}+v \alpha_{g}\right) p \cdot x} \widetilde{S}\left(\alpha_{i}, \mu\right) \text {. } \\
& \left\langle\gamma\left(p, \eta^{*}\right)\left|\bar{q}(x) W_{c}(x, 0) g_{s} \widetilde{G}_{\alpha \beta}(v x) \gamma_{\rho} \gamma_{5} q(0)\right| 0\right\rangle \\
& =-g_{\mathrm{em}} Q_{q} f_{3 \gamma}(\mu) p_{\rho}\left(p_{\beta} \eta_{\alpha}^{*}-p_{\alpha} \eta_{\beta}^{*}\right) \int\left[\mathcal{D} \alpha_{i}\right] e^{i\left(\alpha_{q}+v \alpha_{g}\right) p \cdot x} A\left(\alpha_{i}, \mu\right) \text {. } \\
& \left\langle\gamma\left(p, \eta^{*}\right)\left|\bar{q}(x) W_{c}(x, 0) g_{s} G_{\alpha \beta}(v x) i \gamma_{\rho} q(0)\right| 0\right\rangle \\
& =-g_{\mathrm{em}} Q_{q} f_{3 \gamma}(\mu) p_{\rho}\left(p_{\beta} \eta_{\alpha}^{*}-p_{\alpha} \eta_{\beta}^{*}\right) \int\left[\mathcal{D} \alpha_{i}\right] e^{i\left(\alpha_{q}+v \alpha_{g}\right) p \cdot x} V\left(\alpha_{i}, \mu\right) \text {. } \\
& \left\langle\gamma\left(p, \eta^{*}\right)\left|\bar{q}(x) W_{c}(x, 0) g_{\mathrm{em}} Q_{q} F_{\alpha \beta}(v x) q(0)\right| 0\right\rangle \\
& =-i g_{\mathrm{em}} Q_{q}\langle\bar{q} q\rangle(\mu)\left(p_{\beta} \eta_{\alpha}^{*}-p_{\alpha} \eta_{\beta}^{*}\right) \int\left[\mathcal{D} \alpha_{i}\right] e^{i\left(\alpha_{q}+v \alpha_{g}\right) p \cdot x} S_{\gamma}\left(\alpha_{i}, \mu\right) \text {. } \\
& \left\langle\gamma\left(p, \eta^{*}\right)\left|\bar{q}(x) W_{c}(x, 0) \sigma_{\rho \tau} g_{s} G_{\alpha \beta}(v x) q(0)\right| 0\right\rangle \\
& =g_{\mathrm{em}} Q_{q}\langle\bar{q} q\rangle(\mu)\left[p_{\rho} \eta_{\alpha}^{*} g_{\tau \beta}^{\perp}-p_{\tau} \eta_{\alpha}^{*} g_{\rho \beta}^{\perp}-(\alpha \leftrightarrow \beta)\right] \int\left[\mathcal{D} \alpha_{i}\right] e^{i\left(\alpha_{q}+v \alpha_{g}\right) p \cdot x} T_{1}\left(\alpha_{i}, \mu\right) \\
& +g_{\mathrm{em}} Q_{q}\langle\bar{q} q\rangle(\mu)\left[p_{\alpha} \eta_{\rho}^{*} g_{\tau \beta}^{\perp}-p_{\beta} \eta_{\rho}^{*} g_{\tau \alpha}^{\perp}-(\rho \leftrightarrow \tau)\right] \int\left[\mathcal{D} \alpha_{i}\right] e^{i\left(\alpha_{q}+v \alpha_{g}\right) p \cdot x} T_{2}\left(\alpha_{i}, \mu\right) \\
& +g_{\mathrm{em}} Q_{q}\langle\bar{q} q\rangle(\mu) \frac{\left(p_{\alpha} x_{\beta}-p_{\beta} x_{\alpha}\right)\left(p_{\rho} \eta_{\tau}^{*}-p_{\tau} \eta_{\rho}^{*}\right)}{p \cdot x} \int\left[\mathcal{D} \alpha_{i}\right] e^{i\left(\alpha_{q}+v \alpha_{g}\right) p \cdot x} T_{3}\left(\alpha_{i}, \mu\right) \\
& +g_{\mathrm{em}} Q_{q}\langle\bar{q} q\rangle(\mu) \frac{\left(p_{\rho} x_{\tau}-p_{\tau} x_{\rho}\right)\left(p_{\alpha} \eta_{\beta}^{*}-p_{\beta} \eta_{\alpha}^{*}\right)}{p \cdot x} \int\left[\mathcal{D} \alpha_{i}\right] e^{i\left(\alpha_{q}+v \alpha_{g}\right) p \cdot x} T_{4}\left(\alpha_{i}, \mu\right) .
\end{aligned}
$$




$$
\begin{aligned}
& \left\langle\gamma\left(p, \eta^{*}\right)\left|\bar{q}(x) W_{c}(x, 0) \sigma_{\rho \tau} g_{\mathrm{em}} Q_{q} F_{\alpha \beta}(v x) q(0)\right| 0\right\rangle \\
& =g_{\mathrm{em}} Q_{q}\langle\bar{q} q\rangle(\mu) \frac{\left(p_{\rho} x_{\tau}-p_{\tau} x_{\rho}\right)\left(p_{\alpha} \eta_{\beta}^{*}-p_{\beta} \eta_{\alpha}^{*}\right)}{p \cdot x} \int\left[\mathcal{D} \alpha_{i}\right] e^{i\left(\alpha_{q}+v \alpha_{g}\right) p \cdot x} T_{4}^{\gamma}\left(\alpha_{i}, \mu\right)+\ldots
\end{aligned}
$$

Here, we adopt the following convention for the dual gluon-field strength tensor

$$
\widetilde{G}_{\alpha \beta}=\frac{1}{2} \epsilon_{\alpha \beta \rho \tau} G^{\rho \tau}
$$

Open Access. This article is distributed under the terms of the Creative Commons Attribution License (CC-BY 4.0), which permits any use, distribution and reproduction in any medium, provided the original author(s) and source are credited.

\section{References}

[1] B.L. Ioffe and A.V. Smilga, Nucleon Magnetic Moments and Magnetic Properties of Vacuum in QCD, Nucl. Phys. B 232 (1984) 109 [INSPIRE].

[2] I.I. Balitsky, V.M. Braun and A.V. Kolesnichenko, Radiative Decay $\Sigma^{+} \rightarrow p \gamma$ in Quantum Chromodynamics, Nucl. Phys. B 312 (1989) 509 [InSPIRE].

[3] X.-G. He, T. Li, X.-Q. Li and Y.-M. Wang, PQCD calculation for $\Lambda_{b} \rightarrow \Lambda \gamma$ in the standard model, Phys. Rev. D 74 (2006) 034026 [hep-ph/0606025] [INSPIRE].

[4] T. Mannel and Y.-M. Wang, Heavy-to-light baryonic form factors at large recoil, JHEP 12 (2011) 067 [arXiv:1111.1849] [INSPIRE].

[5] Y.-M. Wang and Y.-L. Shen, Perturbative Corrections to $\Lambda_{b} \rightarrow \Lambda$ Form Factors from QCD Light-Cone Sum Rules, JHEP 02 (2016) 179 [arXiv:1511.09036] [INSPIRE].

[6] Y.-m. Wang, Y. Li and C.-D. Lu, Rare Decays of $\Lambda_{b} \rightarrow \Lambda+\gamma$ and $\Lambda_{b} \rightarrow \Lambda+\ell^{+} \ell^{-}$in the Light-cone Sum Rules, Eur. Phys. J. C 59 (2009) 861 [arXiv:0804.0648] [INSPIRE].

[7] P. Ball and E. Kou, $B \rightarrow \gamma e \nu$ transitions from QCD sum rules on the light cone, JHEP 04 (2003) 029 [hep-ph/0301135] [INSPIRE].

[8] Y.-M. Wang and Y.-L. Shen, Subleading-power corrections to the radiative leptonic $B \rightarrow \gamma \ell \nu$ decay in QCD, JHEP 05 (2018) 184 [arXiv: 1803.06667] [INSPIRE].

[9] A. Khodjamirian, T. Mannel, A.A. Pivovarov and Y.M. Wang, Charm-loop effect in $B \rightarrow K^{(*)} \ell^{+} \ell^{-}$and $B \rightarrow K^{*} \gamma$, JHEP 09 (2010) 089 [arXiv:1006.4945] [INSPIRE].

[10] H.-N. Li, Y.-L. Shen and Y.-M. Wang, Joint resummation for pion wave function and pion transition form factor, JHEP 01 (2014) 004 [arXiv:1310.3672] [INSPIRE].

[11] Y.-M. Wang and Y.-L. Shen, Subleading power corrections to the pion-photon transition form factor in QCD, JHEP 12 (2017) 037 [arXiv: 1706.05680] [INSPIRE].

[12] H.-n. Li, Y.-L. Shen, Y.-M. Wang and H. Zou, Next-to-leading-order correction to pion form factor in $k_{T}$ factorization, Phys. Rev. D 83 (2011) 054029 [arXiv: 1012.4098] [INSPIRE].

[13] A. Czarnecki, W.J. Marciano and A. Vainshtein, Refinements in electroweak contributions to the muon anomalous magnetic moment, Phys. Rev. D 67 (2003) 073006 [Erratum ibid. D 73 (2006) 119901] [hep-ph/0212229] [INSPIRE]. 
[14] V.L. Eletsky and Ya. I. Kogan, Calculation of $D^{*} \rightarrow D \gamma$ and $D^{*} \rightarrow D \pi$ decay widths from QCD sum rules, Z. Phys. C 28 (1985) 155 [InSPIRE].

[15] CLEO collaboration, Measurement of the $D^{*}$ (2010) branching fractions, Phys. Rev. Lett. 69 (1992) 2041 [INSPIRE].

[16] CLEO collaboration, Observation of the radiative decay $D^{*+} \rightarrow D^{+} \gamma$, Phys. Rev. Lett. 80 (1998) 3919 [hep-ex/9711011] [INSPIRE].

[17] BABAR collaboration, Measurement of the branching ratios $\Gamma\left(D_{s}^{*+} \rightarrow D_{s}^{+} \pi^{0}\right) / \Gamma\left(D_{s}^{*+} \rightarrow D_{s}^{+} \gamma\right)$ and $\Gamma\left(D^{* 0} \rightarrow D^{0} \pi^{0}\right) / \Gamma\left(D^{* 0} \rightarrow D^{0} \gamma\right)$, Phys. Rev. D 72 (2005) 091101 [hep-ex/0508039] [INSPIRE].

[18] BESIII collaboration, Precision measurement of the $D^{* 0}$ decay branching fractions, Phys. Rev. D 91 (2015) 031101 [arXiv:1412.4566] [INSPIRE].

[19] A. Yu. Khodjamirian, Dispersion Sum Rules for the Amplitudes of Radiative Transitions in Quarkonium, Phys. Lett. B 90 (1980) 460 [InSPIRE].

[20] T.M. Aliev, E. Iltan and N.K. Pak, Radiative $D^{*}$ meson decays in QCD sum rules, Phys. Lett. B 334 (1994) 169 [INSPIRE].

[21] T.M. Aliev, D.A. Demir, E. Iltan and N.K. Pak, Radiative $B^{*} \rightarrow B \gamma$ and $D^{*} \rightarrow D \gamma$ decays in light cone QCD sum rules, Phys. Rev. D 54 (1996) 857 [hep-ph/9511362] [INSPIRE].

[22] P. Ball, V.M. Braun and N. Kivel, Photon distribution amplitudes in QCD, Nucl. Phys. B 649 (2003) 263 [hep-ph/0207307] [INSPIRE].

[23] J. Rohrwild, Determination of the magnetic susceptibility of the quark condensate using radiative heavy meson decays, JHEP 09 (2007) 073 [arXiv:0708.1405] [INSPIRE].

[24] J.F. Amundson et al., Radiative $D^{*}$ decay using heavy quark and chiral symmetry, Phys. Lett. B 296 (1992) 415 [hep-ph/9209241] [INSPIRE].

[25] P.L. Cho and H. Georgi, Electromagnetic interactions in heavy hadron chiral theory, Phys. Lett. B 296 (1992) 408 [Erratum ibid. B 300 (1993) 410] [hep-ph/9209239] [INSPIRE].

[26] H.-Y. Cheng, C.-Y. Cheung, G.-L. Lin, Y.C. Lin, T.-M. Yan and H.-L. Yu, Chiral Lagrangians for radiative decays of heavy hadrons, Phys. Rev. D 47 (1993) 1030 [hep-ph/9209262] [INSPIRE].

[27] I.W. Stewart, Extraction of the $D^{*} D \pi$ coupling from $D^{*}$ decays, Nucl. Phys. B 529 (1998) 62 [hep-ph/9803227] [INSPIRE].

[28] R. Casalbuoni, A. Deandrea, N. Di Bartolomeo, R. Gatto, F. Feruglio and G. Nardulli, Phenomenology of heavy meson chiral Lagrangians, Phys. Rept. 281 (1997) 145 [hep-ph/9605342] [INSPIRE].

[29] A.V. Manohar and M.B. Wise, Heavy quark physics, Camb. Monogr. Part. Phys. Nucl. Phys. Cosmol. 10 (2000) 1.

[30] B. El-Bennich, M.A. Ivanov and C.D. Roberts, Strong $D^{*} \rightarrow D \pi$ and $B^{*} \rightarrow B \pi$ couplings, Phys. Rev. C 83 (2011) 025205 [arXiv: 1012.5034] [InSPIRE].

[31] ALPHA collaboration, Precision lattice QCD computation of the $B^{*} B \pi$ coupling, Phys. Lett. B 740 (2015) 278 [arXiv: 1404.6951] [INSPIRE].

[32] D. Becirevic and B. Haas, $D^{*} \rightarrow D \pi$ and $D^{*} \rightarrow D \gamma$ decays: Axial coupling and Magnetic moment of $D^{*}$ meson, Eur. Phys. J. C 71 (2011) 1734 [arXiv:0903.2407] [InSPIRE]. 
[33] G.C. Donald, C.T.H. Davies, J. Koponen and G.P. Lepage, Prediction of the $D_{s}^{*}$ width from a calculation of its radiative decay in full lattice QCD, Phys. Rev. Lett. 112 (2014) 212002 [arXiv: 1312.5264] [INSPIRE].

[34] P. Colangelo and A. Khodjamirian, QCD sum rules, a modern perspective, in At the frontier of particle physics. Vol. 3, M. Shifman eds., World Scientific, Singapore (2001) [hep-ph/0010175] [INSPIRE].

[35] P. Colangelo, F. De Fazio and G. Nardulli, Radiative heavy meson transitions, Phys. Lett. B 316 (1993) 555 [hep-ph/9307330] [INSPIRE].

[36] A. Khodjamirian, Form-factors of $\gamma^{*} \rho \rightarrow \pi$ and $\gamma^{*} \gamma \rightarrow \pi^{0}$ transitions and light cone sum rules, Eur. Phys. J. C 6 (1999) 477 [hep-ph/9712451] [INSPIRE].

[37] A. Khodjamirian, T. Mannel and Y.M. Wang, $B \rightarrow K \ell^{+} \ell^{-}$decay at large hadronic recoil, JHEP 02 (2013) 010 [arXiv: 1211.0234] [INSPIRE].

[38] Y.-M. Wang, Factorization and dispersion relations for radiative leptonic B decay, JHEP 09 (2016) 159 [arXiv: 1606.03080] [INSPIRE].

[39] M.J. Dugan and B. Grinstein, On the vanishing of evanescent operators, Phys. Lett. B 256 (1991) 239 [INSPIRE].

[40] S. Herrlich and U. Nierste, Evanescent operators, scheme dependences and double insertions, Nucl. Phys. B 455 (1995) 39 [hep-ph/9412375] [INSPIRE].

[41] M. Beneke and V.A. Smirnov, Asymptotic expansion of Feynman integrals near threshold, Nucl. Phys. B 522 (1998) 321 [hep-ph/9711391] [INSPIRE].

[42] V.A. Smirnov, Applied asymptotic expansions in momenta and masses, Springer Tracts Mod. Phys. 177 (2002) 1 [INSPIRE].

[43] A. Khodjamirian, R. Rückl, S. Weinzierl and O.I. Yakovlev, Perturbative QCD correction to the light cone sum rule for the $B^{*} B \pi$ and $D^{*} D \pi$ couplings, Phys. Lett. B 457 (1999) 245 [hep-ph/9903421] [INSPIRE].

[44] V.M. Belyaev, V.M. Braun, A. Khodjamirian and R. Ruckl, $D^{*} D \pi$ and $B^{*} B \pi$ couplings in QCD, Phys. Rev. D 51 (1995) 6177 [hep-ph/9410280] [INSPIRE].

[45] C.-D. Lü, Y.-L. Shen, Y.-M. Wang and Y.-B. Wei, QCD calculations of $B \rightarrow \pi, K$ form factors with higher-twist corrections, JHEP 01 (2019) 024 [arXiv:1810.00819] [INSPIRE].

[46] J. Gao, C.-D. Lü, Y.-L. Shen, Y.-M. Wang and Y.-B. Wei, Precision calculations of $B \rightarrow V$ form factors in $Q C D$, arXiv: 1907.11092 [INSPIRE].

[47] P. Ball and V.M. Braun, Higher twist distribution amplitudes of vector mesons in QCD: Twist-4 distributions and meson mass corrections, Nucl. Phys. B 543 (1999) 201 [hep-ph/9810475] [INSPIRE].

[48] P. Ball, V.M. Braun and A. Lenz, Twist-4 distribution amplitudes of the $K^{*}$ and $\phi$ mesons in QCD, JHEP 08 (2007) 090 [arXiv:0707.1201] [INSPIRE].

[49] P. Ball and V.M. Braun, Use and misuse of QCD sum rules in heavy to light transitions: The Decay B $\rightarrow$ pev reexamined, Phys. Rev. D 55 (1997) 5561 [hep-ph/9701238] [inSPIRE].

[50] A. Khodjamirian, R. Rückl and C.W. Winhart, The Scalar $B \rightarrow \pi$ and $D \rightarrow \pi$ form-factors in QCD, Phys. Rev. D 58 (1998) 054013 [hep-ph/9802412] [INSPIRE].

[51] V.A. Beilin and A.V. Radyushkin, Quantum Chromodynamic Sum Rules and $J / \psi \rightarrow \eta_{c} \gamma$ Decay, Nucl. Phys. B 260 (1985) 61 [INSPIRE]. 
[52] M. Beneke and D. Yang, Heavy-to-light B meson form-factors at large recoil energy: Spectator-scattering corrections, Nucl. Phys. B 736 (2006) 34 [hep-ph/0508250] [InSPIRE].

[53] M. Beneke and S. Jager, Spectator scattering at NLO in non-leptonic b decays: Tree amplitudes, Nucl. Phys. B 751 (2006) 160 [hep-ph/0512351] [INSPIRE].

[54] G.P. Lepage and S.J. Brodsky, Exclusive Processes in Quantum Chromodynamics: Evolution Equations for Hadronic Wave Functions and the Form-Factors of Mesons, Phys. Lett. B 87 (1979) 359 [INSPIRE].

[55] D. Mueller, Conformal constraints and the evolution of the nonsinglet meson distribution amplitude, Phys. Rev. D 49 (1994) 2525 [INSPIRE].

[56] I.I. Balitsky and V.M. Braun, Evolution Equations for QCD String Operators, Nucl. Phys. B 311 (1989) 541 [INSPIRE].

[57] A. Khodjamirian, T. Mannel, N. Offen and Y.M. Wang, $B \rightarrow \pi \ell \nu_{l}$ Width and $\left|V_{u b}\right|$ from QCD Light-Cone Sum Rules, Phys. Rev. D 83 (2011) 094031 [arXiv:1103.2655] [InSPIRE].

[58] Y.-M. Wang and Y.-L. Shen, QCD corrections to $B \rightarrow \pi$ form factors from light-cone sum rules, Nucl. Phys. B 898 (2015) 563 [arXiv:1506.00667] [inSPIRE].

[59] Particle Data Group collaboration, Review of Particle Physics, Phys. Rev. D 98 (2018) 030001 [INSPIRE].

[60] B. Dehnadi, A.H. Hoang and V. Mateu, Bottom and Charm Mass Determinations with a Convergence Test, JHEP 08 (2015) 155 [arXiv: 1504.07638] [INSPIRE].

[61] M. Beneke, A. Maier, J. Piclum and T. Rauh, The bottom-quark mass from non-relativistic sum rules at NNNLO, Nucl. Phys. B 891 (2015) 42 [arXiv:1411.3132] [INSPIRE].

[62] Flavour Lattice Averaging Group collaboration, FLAG Review 2019, Eur. Phys. J. C 80 (2020) 113 [arXiv:1902.08191] [INSPIRE].

[63] ETM collaboration, Masses and decay constants of $D_{(s)}^{*}$ and $B_{(s)}^{*}$ mesons with $N_{f}=2+1+1$ twisted mass fermions, Phys. Rev. D 96 (2017) 034524 [arXiv:1707.04529] [INSPIRE].

[64] M. Jamin, J.A. Oller and A. Pich, Scalar K $\pi$ form factor and light quark masses, Phys. Rev. D 74 (2006) 074009 [hep-ph/0605095] [INSPIRE].

[65] K.G. Chetyrkin and A. Khodjamirian, Strange quark mass from pseudoscalar sum rule with $O\left(\alpha_{s}^{4}\right)$ accuracy, Eur. Phys. J. C 46 (2006) 721 [hep-ph/0512295] [INSPIRE].

[66] P. Gelhausen, A. Khodjamirian, A.A. Pivovarov and D. Rosenthal, Decay constants of heavy-light vector mesons from QCD sum rules, Phys. Rev. D 88 (2013) 014015 [Erratum ibid. D 89 (2014) 099901] [arXiv: 1305.5432] [INSPIRE].

[67] H. Leutwyler, The Ratios of the light quark masses, Phys. Lett. B 378 (1996) 313 [hep-ph/9602366] [INSPIRE].

[68] S. Ferrara, A.F. Grillo, G. Parisi and R. Gatto, Canonical scaling and conformal invariance, Phys. Lett. B 38 (1972) 333 [inSPIRE].

[69] P. Ball, V.M. Braun, Y. Koike and K. Tanaka, Higher twist distribution amplitudes of vector mesons in QCD: Formalism and twist - three distributions, Nucl. Phys. B 529 (1998) 323 [hep-ph/9802299] [INSPIRE].

[70] A. Khodjamirian, C. Klein, T. Mannel and N. Offen, Semileptonic charm decays $D \rightarrow \pi \ell \nu_{\ell}$ and $D \rightarrow K \ell \nu_{\ell}$ from QCD Light-Cone Sum Rules, Phys. Rev. D 80 (2009) 114005 [arXiv:0907.2842] [INSPIRE]. 
[71] C.-Y. Cheung and C.-W. Hwang, Strong and radiative decays of heavy mesons in a covariant model, JHEP 04 (2014) 177 [arXiv:1401.3917] [INSPIRE].

[72] J.L. Goity and W. Roberts, Radiative transitions in heavy mesons in a relativistic quark model, Phys. Rev. D 64 (2001) 094007 [hep-ph/0012314] [INSPIRE].

[73] E. Bagan, P. Ball, V.M. Braun and H.G. Dosch, QCD sum rules in the effective heavy quark theory, Phys. Lett. B 278 (1992) 457 [inSPIRE].

[74] M. Neubert, Symmetry breaking corrections to meson decay constants in the heavy quark effective theory, Phys. Rev. D 46 (1992) 1076 [InSPIRE].

[75] B. Grinstein and J. Martin Camalich, Weak Decays of Excited B Mesons, Phys. Rev. Lett. 116 (2016) 141801 [arXiv:1509.05049] [INSPIRE].

[76] BABAR collaboration, Measurement of the $D^{*}(2010)^{+}$natural line width and the $D^{*}(2010)^{+}-D^{0}$ mass difference, Phys. Rev. D 88 (2013) 052003 [Erratum ibid. D 88 (2013) 079902] [arXiv: 1304.5009] [INSPIRE].

[77] BABAR collaboration, Measurement of the $D *(2010)^{+}$meson width and the $D *(2010)^{+}-D^{0}$ mass difference, Phys. Rev. Lett. 111 (2013) 111801 [arXiv:1304.5657] [INSPIRE].

[78] QCDSF collaboration, Accelerating the hybrid Monte Carlo algorithm, Phys. Lett. B 564 (2003) 235 [hep-lat/0303026] [INSPIRE].

[79] V.M. Braun, Light cone sum rules, in Progress in heavy quark physics. Proceedings, 4th International Workshop, Rostock Germany (1997), pg. 105 [hep-ph/9801222] [INSPIRE].

[80] A. Khodjamirian, T. Mannel and A.A. Petrov, Direct probes of flavor-changing neutral currents in $e^{+} e^{-}$-collisions, JHEP 11 (2015) 142 [arXiv:1509.07123] [INSPIRE]. 